

\title{
Gentle algebras arising from surface triangulations
}

\author{
Ibrahim Assem, Thomas Brüstle, \\ Gabrielle Charbonneau-Jodoin and Pierre-Guy Plamondon
}

\begin{abstract}
We associate an algebra $A(\Gamma)$ to a triangulation $\Gamma$ of a surface $S$ with a set of boundary marking points. This algebra $A(\Gamma)$ is gentle and Gorenstein of dimension one. We also prove that $A(\Gamma)$ is cluster-tilted if and only if it is cluster-tilted of type $\mathbb{A}$ or $\tilde{A}$, or if and only if the surface $S$ is a disc or an annulus. Moreover all cluster-tilted algebras of type $\mathbb{A}$ or $\tilde{A}$ are obtained in this way.
\end{abstract}

\section{Introduction}

Among the main recent results in the fast-growing theory of cluster algebras is the paper of Fomin, Shapiro and Thurston [Fomin et al. 2008], relating triangulations of oriented surfaces to cluster algebras. This approach, which existed since the beginning of the theory [Caldero et al. 2006], was followed in [Labardini-Fragoso 2009; Schiffler 2008], among others. In the same spirit, we consider in the present paper an unpunctured oriented surface $S$ and a finite set of points $M$, lying on the boundary of $S$ and intersecting every boundary component of $S$. We then associate to a triangulation $\Gamma$ of the marked surface $(S, M)$ a quiver $Q(\Gamma)$, and a potential on $Q(\Gamma)$ (in the sense of [Derksen et al. 2008]), thus defining an algebra $A(\Gamma)$, namely the (noncompleted) Jacobian algebra defined by $Q(\Gamma)$ and the associated potential.

Such an algebra $A(\Gamma)$ has some very nice properties: it is always Gorenstein of dimension one, and also it is a gentle algebra in the sense of [Assem and Skowroński 1987]. In the unpunctured case studied here, our definition coincides with Labardini's definition of a quiver with potential associated to a (possibly punctured) surface [Labardini-Fragoso 2009]. But in the punctured case, one does not

MSC2000: primary 16S99; secondary 16G20, 57N05, 57M50.

Keywords: bordered surface with marked points, triangulated surface, quiver with potential, gentle algebra.

Assem is partially supported by NSERC of Canada and the Université de Sherbrooke. Charbonneau was working under a summer research fellowship of NSERC. Brüstle is partially supported by NSERC, by Bishop's University and the Université de Sherbrooke. Plamondon was supported by an NSERC graduate fellowship. 
get gentle algebras, or even string algebras. For instance, a once-punctured disc gives rise to cluster-tilted algebras of type $\mathbb{D}$ [Schiffler 2008].

Gentle algebras form a particularly nice subclass of the class of string algebras of [Butler and Ringel 1987] and are much investigated in the representation theory of algebras. For instance, this subclass contains the tilted algebras of type $\mathbb{A}$ and $\widetilde{A}$ (see [Assem 1982] and [Assem and Skowroński 1987], respectively) and it is closed under tilting and even under derived equivalence (see [Schröer 1999] and [Schröer and Zimmermann 2003], respectively).

Our objective in this paper is twofold. Firstly, we ask which gentle algebras arise in this way, that is, are induced from triangulations of an unpunctured surface with boundary marked points. We show in Proposition 2.8 that this is the case for every gentle algebra such that every relation lies on what we call a 3-cycle with radical-square zero (see definition before Theorem 2.7 or [Buan and Vatne 2008] for the definition). Secondly, we ask which gentle algebras are cluster-tilted. The class of cluster-tilted algebras, introduced in [Buan et al. 2007], has been much investigated and is by now well-understood (see, for instance, [Assem et al. 2008a; Barot et al. 2008; Buan et al. 2006; Buan and Vatne 2008; Caldero et al. 2006; Keller 2009; Keller and Reiten 2007; Schiffler 2008]). In particular, it was shown in [Assem et al. 2008a] that every cluster-tilted algebra is the relation-extension of a tilted algebra, that is, it is the trivial extension of a tilted algebra $C$ by the $C$-C-bimodule $\operatorname{Ext}_{C}^{2}(D C, C)$. We may now state the main result of this paper.

Theorem 1.1. Let $A(\Gamma)$ be the algebra associated to the triangulation $\Gamma$ of an unpunctured marked surface $(S, M)$. Then the following statements are equivalent:

(1) $A(\Gamma)$ is cluster-tilted.

(2) $A(\Gamma)$ is cluster-tilted of type $\mathbb{A}$ and $\widetilde{\mathbb{A}}$.

(3) $A(\Gamma)$ is the relation-extension of a tilted algebra of type $\mathbb{A}$ and $\widetilde{\mathbb{A}}$.

(4) The surface $S$ is a disc or an annulus.

Moreover, all cluster-tilted algebras of type $\mathbb{A}($ or $\widetilde{\mathbb{A}})$ are of the form $A(\Gamma)$ for some triangulation of a disc $S$ (or an annulus $S$, respectively).

Actually, we prove in Theorem 3.3 that a cluster-tilted algebra is gentle if and only if it is of type $\mathbb{A}$ and $\widetilde{A}$, or if and only if it is the relation-extension of a gentle tilted algebra, and the latter coincide with the tilted algebras of type $\mathbb{A}$ or $\widetilde{\mathbb{A}}$, respectively.

The case where $S$ is a disc has already been studied in [Caldero et al. 2006], and it is known that the bound quivers of all cluster-tilted algebras of type $\mathbb{A}$ arise from triangulations of the (unpunctured) disc. These algebras have also been described explicitly in [Buan and Vatne 2008]. Also, the potential we use for defining the cluster-tilted algebras of type $\widetilde{A}$ is a particular case of the potential recently defined 
by Keller [2009]. However, we do not use this fact, but rather present another proof (predating Keller's result), which uses [Assem et al. 2008a] and properties of the second extension group.

The paper is organised as follows: in Section 2, we define our algebras $A(\Gamma)$ and prove their main properties in Theorem 2.7. Section 3 is devoted to the classification of the gentle cluster-titled algebras and Section 4 to the proof of our main theorem and some of its consequences. We conclude with an example of an algebra $A(\Gamma)$ that is not of polynomial growth in the sense of [Skowroński 1990].

\section{Algebras arising from surface triangulations}

Throughout this paper, the algebras we consider are basic connected algebras over a fixed algebraically closed field $k$. Unless otherwise stated, all algebras are finitedimensional. Consequently, they are given in the form $A=k Q / I$ where $Q$ is a quiver and $I$ is an admissible ideal of the path algebra $k Q$ [Assem et al. 2006]. The pair $(Q, I)$ is called a bound quiver, and the algebra $A=k Q / I$ is referred to as a bound quiver algebra.

Given a bound quiver algebra $A=k Q / I$, for every vertex $x$ of $Q$ we denote by $e_{x}$ the idempotent of $A$ associated to $x$. Also, $P_{x}, I_{x}$ and $S_{x}$ will be the corresponding indecomposable projective module, indecomposable injective module and simple module, respectively.

We study in this section the algebra associated with a surface triangulation. For background material on oriented surfaces we refer to [Massey 1991].

The medial quiver $\boldsymbol{Q}(\boldsymbol{\Gamma})$. We first recall from [Fomin et al. 2008] the construction of a quiver for every triangulation of a marked surface. Let $S$ be an oriented surface with boundary $\partial S$, and let $M$ be a nonempty finite set of points on $\partial S$ intersecting each connected component of the boundary $\partial S$. In this paper, we only consider the case where there are no punctures, that is, we request that the set of marked points $M$ be contained in the boundary $\partial S$. The pair $(S, M)$ is referred to as an unpunctured bordered surface with marked points.

An $\operatorname{arc}$ in $(S, M)$ is a curve $\gamma$ in $S$ such that:

- The endpoints of $\gamma$ are marked points in $M$.

- $\gamma$ does not intersect itself, except that its endpoints may coincide.

- $\gamma$ intersects the boundary of $\partial S$ only in its endpoints.

- $\gamma$ does not cut out a monogon (that is, $\gamma$ is not contractible into a point of $M$ ).

We call an arc $\gamma$ a boundary arc if it cuts out a digon (that is, $\gamma$ is homotopic to a curve $\delta$ on the boundary $\partial S$ that intersects $M$ only in its endpoints). Otherwise, $\gamma$ is said to be an internal arc. Each arc $\gamma$ is considered up to homotopy in the 
class of such curves. A triangulation of $(S, M)$ is a maximal collection $\Gamma$ of arcs that do not intersect in the interior of $S$ (more precisely, there are curves in their respective homotopy classes that do not intersect in the interior of $S$ ).

Proposition 2.1 [Fomin et al. 2008, (2.10)]. In each triangulation of $(S, M)$, the number of internal arcs is

$$
n=6 g+3 b+c-6,
$$

where $g$ is the genus of $S, b$ is the number of boundary components, and $c=|M|$ is the number of marked points.

This proposition also indicates that in some cases a triangulation does not exist (for instance a disc with one marked point would give $n=-2$ ). We consider from now on only marked surfaces $(S, M)$ that admit a triangulation. Given a triangulation $\Gamma$, we also refer to $M$ as the set of vertices of $\Gamma$. The triangles are the components of $S \backslash \Gamma$ with the arcs of $\Gamma$ as edges.

We denote by $Q(\Gamma)$ the medial quiver of internal arcs of $\Gamma$. That is, $Q(\Gamma)$ is the quiver whose set of points is the set of internal $\operatorname{arcs}$ of $\Gamma$, and the arrows are defined as follows: whenever there is a triangle $T$ in $\Gamma$ containing two internal arcs $a$ and $b$, then $Q(\Gamma)$ contains an arrow $a \rightarrow b$ if $a$ is a predecessor of $b$ with respect to clockwise orientation at the joint vertex of $a$ and $b$ in $T$ (we can talk about clockwise orientation around each marked point because $S$ is an oriented surface).

Example. We illustrate the construction of $Q(\Gamma)$ when $\Gamma$ is a triangulation of an octagon:
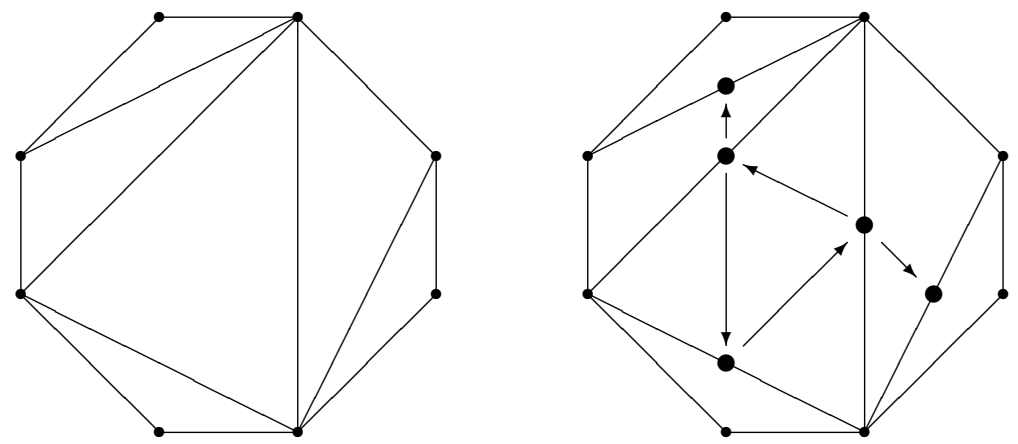

Lemma 2.2. The quiver $Q(\Gamma)$ contains no oriented cycles of length $\leq 2$.

Proof. We first show that $Q(\Gamma)$ contains no loops. A loop $\alpha$ at the point $a$ of $Q(\Gamma)$ would arise from a triangle $T$ in $\Gamma$ in the following way:

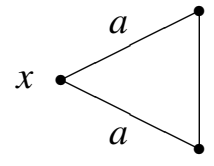

gives rise to

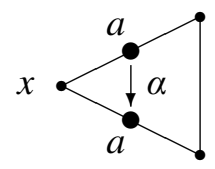


But in this case the triangle $T$ is homeomorphic to

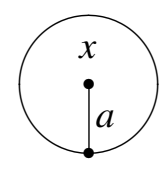

which means that $x$ is an internal vertex, contradicting our assumption that $M$ is contained in the boundary of $S$.

We now show that $Q(\Gamma)$ contains no oriented cycles of length two. Indeed, such a cycle corresponds to the following situation in $\Gamma$ :

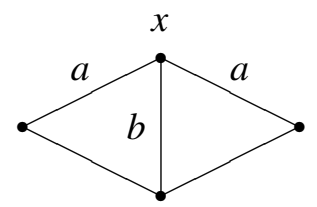

Then a neighbourhood of $x$ is homeomorphic to

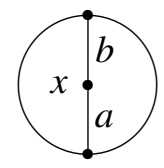

which again contradicts the assumption that $\Gamma$ contains no internal vertices.

Remark. Fomin et al. [2008] associate a skew-symmetric matrix $B(\Gamma)$ to a triangulation $\Gamma$ of $(S, M)$. This construction is equivalent to the construction of the quiver $Q(\Gamma)$ we consider here. Since $Q(\Gamma)$ contains no oriented cycles of length $\leq 2$, it is uniquely determined by a skew-symmetric matrix $B$ (where the number of arrows between two vertices is given by the entries of $B$, and the direction of the arrows is determined by the sign of the matrix entries). It is easy to see that $B$ coincides with $B(\Gamma)$. Thus all the results from [Fomin et al. 2008] apply; in particular, mutations of the quiver $Q(\Gamma)$ correspond to flips of the triangulation $\Gamma$.

Let $b$ be an internal arc of $\Gamma$. Thus $b$ is one diagonal of the quadrilateral formed by the two triangles of $\Gamma$ that contain $b$. The flip of $b$ replaces the edge $b$ by the other diagonal $b^{*}$ of the same quadrilateral. Keeping all other edges unchanged, one obtains a new triangulation $\mu_{b}(\Gamma)$.
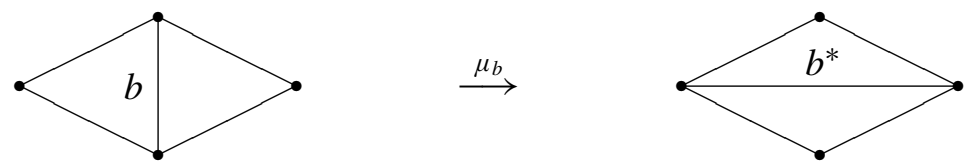

An essential ingredient in the definition of cluster algebras by Fomin and Zelevinsky [2002] is the mutation of skew-symmetric matrices. Reformulated in the language of quivers, one obtains a mutation of quivers $Q \mapsto \mu_{b}(Q)$. The following proposition shows that flips of the triangulation commute with quiver mutations. 
Proposition 2.3 [Fomin et al. 2008, Proposition 4.8]. Suppose that the triangulation $\mu_{b}(\Gamma)$ is obtained from $\Gamma$ by a flip replacing the diagonal labelled $b$. Then

$$
Q\left(\mu_{b}(\Gamma)\right)=\mu_{b}(Q(\Gamma))
$$

The algebra $\boldsymbol{A}(\boldsymbol{\Gamma})$. We define in this section an algebra $A(\Gamma)$ for each triangulation $\Gamma$ of the unpunctured marked surface $(S, M)$. Our construction generalizes the one given in [Caldero et al. 2006] for polygons. An even more general case is considered in [Labardini-Fragoso 2009], where such an algebra $A(\Gamma)$ is defined for a general marked surface (allowing punctures). If there are no punctures, the definitions coincide (although Labardini works in the equivalent framework of opposite medial quivers).

A triangle $T$ in $\Gamma$ is called an internal triangle if all edges of $T$ are internal arcs. Every internal triangle $T$ in $\Gamma$ gives rise to an oriented cycle $\alpha_{T} \beta_{T} \gamma_{T}$ in $Q(\Gamma)$, unique up to cyclic permutation of the factors $\alpha_{T}, \beta_{T}, \gamma_{T}$. We define

$$
W=\sum_{T} \alpha_{T} \beta_{T} \gamma_{T}
$$

where the sum runs over all internal triangles $T$ of $\Gamma$. Then $W$ is a potential on $Q(\Gamma)$ and we define $A(\Gamma)$ to be the (noncompleted) Jacobian algebra of $(Q, W)$ [Derksen et al. 2008; Keller 2007]. Thus $A(\Gamma)$ can be described as a quotient $A(\Gamma)=k Q(\Gamma) / I(\Gamma)$ of the path algebra $k Q(\Gamma)$ by the ideal $I(\Gamma)$ generated by all paths $\alpha_{T} \beta_{T}, \beta_{T} \gamma_{T}$ and $\gamma_{T} \alpha_{T}$ whenever $T$ is an internal triangle of $\Gamma$. Labardini [2009] showed that flips in the triangulation correspond to mutations of the quiver with potential $(Q(\Gamma), W)$ as defined in [Derksen et al. 2008].

The following result is shown in [Labardini-Fragoso 2009, Theorem 36] for the more general case of punctured marked surfaces.

Lemma 2.4. Let $\Gamma$ be a triangulation of an unpunctured marked surface $(S, M)$. Then the algebra $A(\Gamma)$ is finite-dimensional.

We show in Lemma 2.5 that the algebras $A(\Gamma)$ belong to a class of algebras called gentle algebras. Recall from [Assem and Skowroński 1987] that a finitedimensional algebra is gentle if it admits a presentation $A=k Q / I$ satisfying the following conditions:

(G1) At each point of $Q$ start at most two arrows and stop at most two arrows.

(G2) The ideal $I$ is generated by paths of length 2 .

(G3) For each arrow $\beta$ there is at most one arrow $\alpha$ and at most one arrow $\gamma$ such that $\alpha \beta \in I$ and $\beta \gamma \in I$.

(G4) For each arrow $\beta$ there is at most one arrow $\alpha$ and at most one arrow $\gamma$ such that $\alpha \beta \notin I$ and $\beta \gamma \notin I$. 
If the pair $(Q, I)$ satisfies conditions (G1) through (G4), we call it a gentle bound quiver, or a gentle presentation of $A=k Q / I$. Note that in contrast to [Assem and Skowroński 1987], we do not assume that $A=k Q / I$ is triangular. An algebra $A=k Q / I$ where $I$ is generated by paths and $(Q, I)$ satisfies the two conditions (G1) and (G4) is called a string algebra [Butler and Ringel 1987], and thus every gentle algebra is a string algebra. The gentle algebras can be characterized by the fact that their repetitive categories are special biserial [Assem and Skowroński 1987; Pogorzały and Skowroński 1991].

We recall here the classification of indecomposable modules over a string algebra $A=k Q / I$ which is given in [Butler and Ringel 1987] in terms of reduced walks in the quiver $Q$. A string in $A$ is by definition a reduced walk $w$ in $Q$ avoiding the zero-relations, and thus $w$ is a sequence

$$
w=x_{1} \stackrel{\alpha_{1}}{\longleftrightarrow} x_{2} \stackrel{\alpha_{1}}{\longleftrightarrow} \cdots \stackrel{\alpha_{n-1}}{\longleftrightarrow} x_{n},
$$

where the $x_{i}$ are vertices of $Q$ and each $\alpha_{i}$ is an arrow between the vertices $x_{i}$ and $x_{i+1}$ in either direction such that $w$ does not contain a sequence of the form

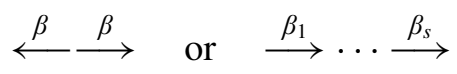

with $\beta_{1} \cdots \beta_{s} \in I$, or their duals. A string is cyclic if the first and the last vertex coincide. A band is defined to be a cyclic string $b$ such that each power $b^{n}$ is a string, but $b$ itself is not a proper power of some string $c$.

The string module $M(w)$ is obtained from the string $w$ by replacing each $x_{i}$ in $w$ by a copy of the field $k$. The action of an arrow $\alpha$ on $M(w)$ is induced by the relevant identity morphisms if $\alpha$ lies on $w$, and is zero otherwise. The dimension vector $\operatorname{dim} M(w)$ of $M(w)$ is obtained by counting how often the string $w$ passes through each vertex $x$ of the quiver $Q$ :

$$
\underline{\operatorname{dim}} M(w)=\left(\sum_{1 \leq i \leq n} \delta_{x, x_{i}}\right)_{x \in Q_{0}},
$$

where $\delta_{x, x_{i}}=1$ for $x=x_{i}$ and $\delta_{x, x_{i}}=0$ otherwise. Similarly, each band $b$ in $A$ gives rise to a family of band modules $M(b, \lambda, n)$ where $\lambda \in k$ and $n \in \mathbb{N}$ (we refer to [Butler and Ringel 1987] for the precise definition). All string and band modules are indecomposable, and in fact every indecomposable $A$-module is either a string module $M(w)$ or a band module $M(b, \lambda, n)$ [Butler and Ringel 1987].

We now return to the study of algebras stemming from surface triangulations:

Lemma 2.5. Let $\Gamma$ be a triangulation of an unpunctured marked surface $(S, M)$. Then $A(\Gamma)$ is a gentle algebra.

Proof. By Lemma 2.4, the algebra $A(\Gamma)$ is finite-dimensional, so we only need to verify conditions $(\mathrm{G} 1)$ to $(\mathrm{G} 4)$ for the bound quiver $(Q(\Gamma), I(\Gamma))$ of $A(\Gamma)$. 
(G2): By definition, the ideal $I(\Gamma)$ is generated by paths of length two.

(G1): Let $a$ be a point of $Q(\Gamma)$ corresponding to an internal $\operatorname{arc} a$ of $\Gamma$. Since $\Gamma$ is a triangulation of a surface, the arc $a$ is contained in at most two triangles:

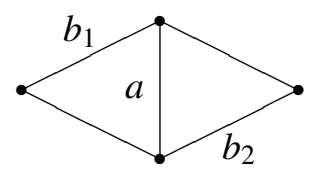

Hence there are at most two arrows $\alpha_{1}: b_{1} \rightarrow a$ and $\alpha_{2}: b_{2} \rightarrow a$ of $Q(\Gamma)$ ending in $a$. The same holds for arrows starting in a point $a$.

(G3), (G4): Suppose now that $Q(\Gamma)$ contains $\alpha_{1}, \alpha_{2}, \beta$ as follows:

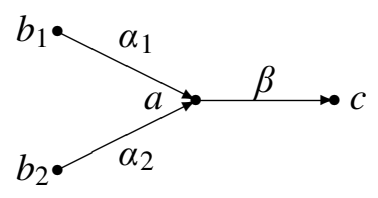

We have to show that precisely one of $\alpha_{1} \beta, \alpha_{2} \beta$ belongs to $I(\Gamma)$. In $\Gamma$, the internal arcs $a, b_{1}, b_{2}$ belong to two triangles as considered in the proof of (G1). The arrow $\beta$ encodes that the $\operatorname{arc} c$ is a successor of $a$ in one of these triangles, say the one formed by $a, b_{1}, c$. This gives rise to the relation $\alpha_{1} \beta$, and $\alpha_{2} \beta$ does not belong to $I(\Gamma)$ since $\alpha_{2}$ and $\beta$ arise from different triangles.

From the construction of $A(\Gamma)$ it is clear that for each $\alpha \beta \in I(\Gamma)$ there is an arrow $\gamma$ in $Q(\Gamma)$ such that $\beta \gamma \in I(\Gamma)$ and $\gamma \alpha \in I(\Gamma)$. In the following lemma we study a homological property of all gentle algebras satisfying this condition: an algebra $A$ is Gorenstein of dimension one if the injective dimension of the (finitely generated) projective $A$-modules is at most one, and the projective dimension of the (finitely generated) injective $A$-modules is at most one. Note that all clustertilted algebras are Gorenstein of dimension one, and that an algebra of Gorenstein dimension one is either hereditary or has infinite global dimension; see [Keller and Reiten 2007].

Lemma 2.6. Let $A=k Q / I$ be a gentle algebra such that for each $\alpha \beta \in I$ there is an arrow $\gamma$ in $Q$ such that $\beta \gamma \in I$ and $\gamma \alpha \in I$. Then $A$ is Gorenstein of dimension one.

Proof. We only compute the projective dimension of the injective modules here; the proof of the other part in the definition of Gorenstein of dimension one is dual. It is sufficient to show that for every vertex $x$ of $Q$ the corresponding indecomposable injective $A$-module $I_{x}$ has projective dimension at most one. To do so, we construct explicitly a projective resolution of $I_{x}$. We write the string module $I_{x}$ as $I_{x}=$ $M\left(u_{1} \alpha_{1} \alpha_{2}^{-1} u_{2}^{-1}\right)$, where $u_{1}$ and $u_{2}$ are oriented paths. Both paths might have length 
zero, and in this case, also the arrows $\alpha_{1}$ and $\alpha_{2}$ might not be present. The following figure is used throughout the proof:

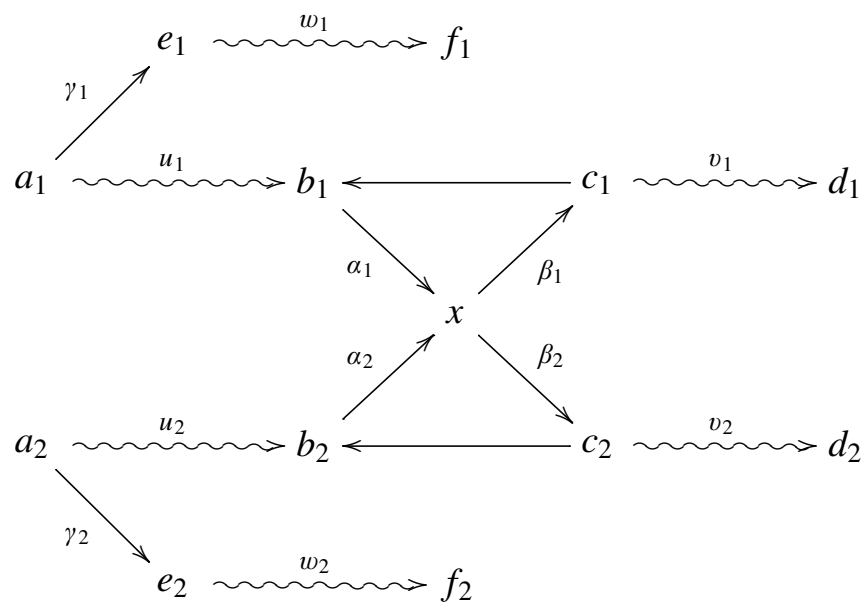

Note that $\left\{x, c_{1}, b_{1}\right\}$ and $\left\{x, c_{2}, b_{2}\right\}$ form oriented cycles in $Q$ such that the composition of any two consecutive arrows is zero. Let

$$
p_{0}: P(0) \rightarrow I_{x}
$$

be a projective cover; then

$$
P(0)=M\left(w_{1}^{-1} \gamma_{1}^{-1} u_{1} \alpha_{1} \beta_{2} v_{2}\right) \oplus M\left(w_{2}^{-1} \gamma_{2}^{-1} u_{2} \alpha_{2} \beta_{1} v_{1}\right)
$$

and

$$
\text { Ker } p_{0}=M\left(w_{1}\right) \oplus M\left(w_{2}\right) \oplus M\left(v_{1}^{-1} \beta_{1}^{-1} \beta_{2} v_{2}\right)
$$

(note that some summands of the terms of this sequence can be zero). We show that $\operatorname{Ker} p_{0}$ is projective, thus obtaining the desired projective resolution

$$
0 \longrightarrow \operatorname{Ker} p_{0} \longrightarrow P(0) \stackrel{p_{0}}{\longrightarrow} I_{x} \longrightarrow 0 .
$$

In order to see that the first two summands of Ker $p_{0}$ are projective (namely the indecomposable projectives $P_{e_{1}}$ and $P_{e_{2}}$ ), one has to show that there are no other arrows starting at the vertices $e_{1}, e_{2}$. Suppose there is an arrow $\delta_{1}: e_{1} \rightarrow y$ in $Q$. Since the algebra $A$ is gentle, the composition $\gamma_{1} \delta_{1}$ lies in the ideal $I$. The assumption of the lemma guarantees the existence of a cycle $\gamma_{1} \delta_{1} \epsilon_{1}$ such that $\gamma_{1} \delta_{1}, \delta_{1} \epsilon_{1}, \epsilon_{1} \gamma_{1} \in I$. But then the simple $A$-module $S_{y}$ would be a composition factor of $I_{x}$, contradicting the assumption $I_{x}=M\left(u_{1} \alpha_{1} \alpha_{2}^{-1} u_{2}^{-1}\right)$. This shows that $M\left(w_{1}\right)=P_{e_{1}}$, and a similar argument shows that $M\left(w_{2}\right)=P_{e_{2}}$. Since $M\left(v_{1}^{-1} \beta_{1}^{-1} \beta_{2} v_{2}\right)=P_{x}$, we conclude that Ker $p_{0}$ is projective.

Example. We illustrate the projective resolution constructed in Lemma 2.6 when $\Gamma$ is the following triangulation of a polygon with 11 vertices (where the midpoints 
of internal arcs are labeled):

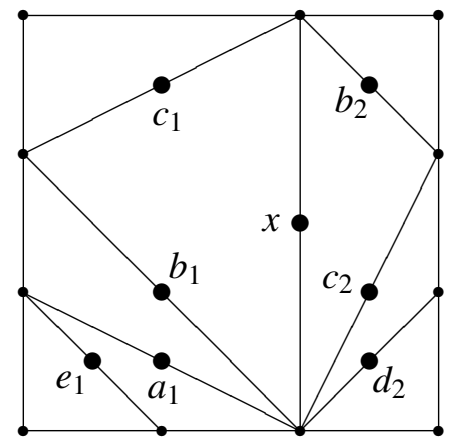

The corresponding algebra $A(\Gamma)$ is given by the quiver

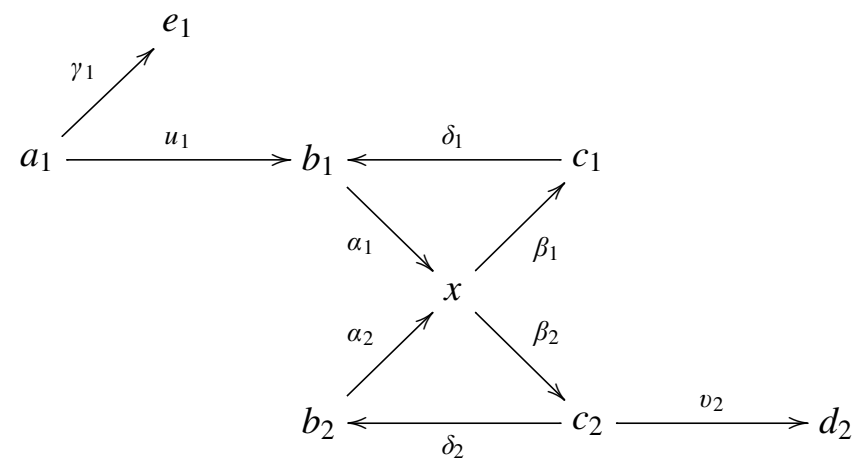

with relations $\alpha_{1} \beta_{1}=\beta_{1} \delta_{1}=\delta_{1} \alpha_{1}=0$ and $\alpha_{2} \beta_{2}=\beta_{2} \delta_{2}=\delta_{2} \alpha_{2}=0$. The projective resolution of the injective module $I_{x}$ is then

$$
0 \longrightarrow P_{e_{1}} \oplus P_{x} \longrightarrow P_{a_{1}} \oplus P_{b_{2}} \longrightarrow I_{x} \longrightarrow 0,
$$

where $P_{e_{1}}$ is simple and $P_{x}=M\left(\beta_{1}^{-1} \beta_{2} v_{2}\right), P_{a_{1}}=M\left(u_{1} \alpha_{1} \beta_{2} v_{2}\right)$ and $P_{b_{2}}=$ $M\left(\alpha_{2} \beta_{1}\right)$.

We recall from [Gabriel 1981] the concept of Galois coverings of bound quiver algebras: Let $\Lambda=k \tilde{Q} / \tilde{I}$ be a bound quiver algebra (where the quiver $\tilde{Q}$ is not necessarily finite). A group $G$ of $k$-linear automorphisms of $\Lambda$ is acting freely on $\Lambda$ if $g e_{x} \neq e_{x}$ for each vertex $x$ of $\tilde{Q}$ and each $g \neq 1$ in $G$. In this case the multiplication in $\Lambda$ induces a multiplication on the set $\Lambda / G$ of $G$-orbits which turns $\Lambda / G$ into an algebra. The canonical projection $\Lambda \rightarrow \Lambda / G$ is called the Galois covering of $\Lambda / G$ with group $G$.

In the following theorem we call (as in [Buan and Vatne 2008]) a 3-cycle an oriented cycle $\alpha \beta \gamma$ where $\alpha, \beta, \gamma$ are three distinct arrows; and by a 3-cycle with radical square zero we mean a 3-cycle $\alpha \beta \gamma$ in an algebra $k Q / I$ such that $\alpha \beta, \beta \gamma, \gamma \alpha$ 
all lie in $I$. By a simple cycle we refer to a subquiver $C$ of $Q$ with $n$ distinct vertices $\left\{x_{0}, x_{1}, \ldots, x_{n-1}, x_{n}=x_{0}\right\}$ and $n$ arrows $\alpha_{i}: x_{i} \rightarrow x_{i+1}$, for $i=1, \ldots, n-1$.

Theorem 2.7. Let $\Gamma$ be a triangulation of an unpunctured marked surface $(S, M)$.

(1) The algebra $A(\Gamma)$ is a gentle algebra.

(2) The algebra $A(\Gamma)$ is Gorenstein of dimension one.

(3) There is a relation in $A(\Gamma)$ from $x$ to $y$ only if there is an arrow $y \rightarrow x$.

(4) $A(\Gamma)$ admits a Galois covering by a bound quiver algebra $k \tilde{Q} / \tilde{I}$ satisfying:

(T1) Every simple cycle in $\tilde{Q}$ is a 3-cycle with radical square zero.

(T2) The only relations in $\tilde{I}$ are those in the 3-cycles.

Proof. Part (1) is shown in Lemma 2.5, and part (2) is shown in Lemma 2.6 since the condition imposed on the gentle algebra $A$ there clearly holds for the algebra $A(\Gamma)$. Part (3) follows directly from the definition of $A(\Gamma)$. Maybe the most intuitive way to obtain the Galois covering required in part (4) is the following. By construction, the only relations in the algebra $A(\Gamma)$ are those in the 3-cycles. In a first step, we identify all 3-cycles to points, replacing each 3-cycle $C$ with vertices $\left\{x_{1}, x_{2}, x_{3}\right\}$ by one single vertex $x$ and replacing each arrow $y \rightarrow x_{i}$ (or $x_{i} \rightarrow y$, respectively) by an arrow $y \rightarrow x$ (or $x \rightarrow y$, respectively). The quiver $\bar{Q}$ thus obtained contains no relations, and we let $\widetilde{\bar{Q}}$ be its universal Galois covering, a (maybe infinite) tree. The bound quiver $(\tilde{Q}, \tilde{I})$ is then obtained by placing back the 3 -cycles $C=\left\{x_{1}, x_{2}, x_{3}\right\}$ for all contracted vertices $x$ of $\widetilde{\bar{Q}}$.

Note that the finite quivers satisfying conditions (T1) and (T2) from the previous theorem form precisely the class of quivers $2_{n}$ considered in [Buan and Vatne 2008], where also the same relations are imposed. It would be interesting to relate the Galois covering $(\tilde{Q}, \tilde{I})$ constructed above with the universal cover of the bordered surface $(S, M)$.

Recovering topological data from $\boldsymbol{A}(\boldsymbol{\Gamma})$. The condition (4) in Theorem 2.7 is very strong. Combined with the fact that the algebra is gentle, it implies the remaining conditions (2) and (3). We show in this section that a gentle algebra satisfying condition (4) is given by an unpunctured marked surface.

First we give a different combinatorial description of the algebras studied here. Consider the following two bound quivers, where type $I$ is a quiver of type $\mathbb{A}_{2}$, and type II is a 3-cycle with radical square zero:

\section{Type I $\longrightarrow$ Type II}

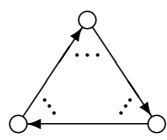

Using these bound quivers one can construct algebras in the following way. Suppose we start with a collection $C$ of disjoint blocks of types I and II. Choose 
a partial matching (that is to say a partial bijection) $\pi$ of the vertices in $C$, where matching a vertex to another vertex of the same block is not allowed. Identifying (or "gluing") the vertices within each pair of the matching we obtain an algebra $A(C, \pi)$. Note that the arrows are not identified by this procedure, so one might obtain parallel arrows or two-cycles. We consider only matchings where the algebra $A(C, \pi)$ is connected.

The procedure of gluing blocks is considered in a more general situation (using plenty of building blocks) in [Brüstle 2001], where the resulting algebras are called kit algebras. A similar construction to glue blocks of type I, II and four more types is described in [Fomin et al. 2008].

We show below that the gentle algebras that admit a Galois covering satisfying conditions (T1) and (T2) from Theorem 2.7 are algebras of the form $A(C, \pi)$, and thus results from [Fomin et al. 2008] concerning these algebras can be applied.

Proposition 2.8. Let $A=k Q / I$ be a gentle algebra where every relation lies on a 3-cycle with radical square zero. Then there exists an unpunctured marked surface $(S, M)$ with a triangulation $\Gamma$ such that $A(\Gamma)=A$.

Proof. The statement follows from [Fomin et al. 2008, (14.1)] once we show that the algebra $A$ admits a unique block decomposition $A=A(C, \pi)$ using blocks of types I and II. We therefore define $C$ to be the disjoint union of all 3-cycles with radical square zero of $A$ together with the disjoint union of all remaining arrows from $A$. Denote by $f$ the quiver morphism $f: C \rightarrow Q$ that identifies the blocks of $C$ with their images in $Q$.

We first show that $\left|f^{-1}(x)\right| \leq 2$ for each vertex $x \in Q$. Indeed, if $f^{-1}(x)$ contains three different vertices, then there are three different arrows in $Q$ adjacent to the vertex $x$. But since the algebra $A$ is gentle, there has to be one relation between these three arrows. However, the set $C$ is constructed in such a way that all relations of $A$ belong to one of the components in $C$, so there are no relations between arrows corresponding to different components of $C$, and so the fiber $f^{-1}(x)$ contains at most two vertices.

We now define a matching $\pi$ on $C$ relating $x_{1}$ to $x_{2}$ whenever $f^{-1}(x)=\left\{x_{1}, x_{2}\right\}$. As required in the definition of $A(C, \pi)$, we do not match a vertex to itself or to some vertex in the same block. It is clear from the construction that $A=A(C, \pi)$. Moreover, the choice of blocks of type I or II is unique since all relations have to correspond to a block of type II.

We would like to point out that all algebras $A(\Gamma)$ given by a triangulation $\Gamma$ of an unpunctured marked surface are of the form $A(C, \pi)$ for some $C$ and $\pi$, but the converse is not true: One can easily produce two-cycles in an algebra $A(C, \pi)$, but this never occurs for the algebras $A(\Gamma)$ as we have shown in Lemma 2.2. 


\section{Gentle cluster-tilted algebras}

Cluster-tilted algebras. Let $\Delta$ be an acyclic quiver. In [Buan et al. 2006] the cluster category $\mathscr{C}_{\Delta}$ is studied in order to obtain a categorical interpretation of the cluster variables of the cluster algebra associated with $\Delta$. It is shown there that clusters correspond bijectively to tilting objects $T$ in $\mathscr{C}_{\Delta}$. Their endomorphism rings $\operatorname{End}_{C_{\Delta}}(T)$ are called cluster-tilted algebras of type $\Delta$. They were introduced and studied in [Buan et al. 2007].

Here we use a different description that has been given in [Assem et al. 2008a]. Let $A$ be the hereditary algebra $k \Delta$. An $A$-module $T$ is called a tilting module if $\operatorname{Ext}_{A}^{1}(T, T)=0$ and the number of isomorphism classes of indecomposable summands of $T$ equals the number of isomorphism classes of simple $A$-modules. In this case, the endomorphism ring $\operatorname{End}_{A}(T)$ is called a tilted algebra of type $\Delta$.

Let $C$ be an algebra of global dimension two. The trivial extension

$$
\tilde{C}=C \ltimes \operatorname{Ext}_{C}^{2}(D C, C)
$$

of $C$ by the $C$ - $C$-bimodule $\operatorname{Ext}_{C}^{2}(D C, C)$ is called the relation-extension of $C$. It is useful to describe explicitly the operations on $\tilde{C}$. As an abelian group, $\tilde{C}=$ $C \oplus \operatorname{Ext}_{C}^{2}(D C, C)$. Therefore, let $(c, \boldsymbol{e})$ and $\left(c^{\prime}, \boldsymbol{e}^{\prime}\right)$ be two elements of $\tilde{C}$, where $\boldsymbol{e}$ and $\boldsymbol{e}^{\prime}$ are respectively represented by the exact sequences of $C$-modules

$$
\begin{array}{ll}
\boldsymbol{e}: & 0 \longrightarrow P \longrightarrow M \longrightarrow N \longrightarrow{ }^{\longrightarrow} \longrightarrow{ }^{\longrightarrow} \longrightarrow{ }^{\prime}, \\
\boldsymbol{e}^{\prime}: & 0 \longrightarrow M^{\prime} \longrightarrow N^{\prime} \longrightarrow I^{\prime} \longrightarrow 0,
\end{array}
$$

with $P, P^{\prime}$ projective and $I, I^{\prime}$ injective. The addition is given by

$$
(c, \boldsymbol{e})+\left(c^{\prime}, \boldsymbol{e}^{\prime}\right)=\left(c+c^{\prime}, \boldsymbol{e}+\boldsymbol{e}^{\prime}\right),
$$

where the sum $c+c^{\prime}$ is the ordinary sum inside $C$, while $\boldsymbol{e}+\boldsymbol{e}^{\prime}$ is the Baer sum in $\operatorname{Ext}_{C}^{2}(D C, C)$ (for which we refer to any textbook of homological algebra). The product in $\tilde{C}$ is given by the formula

$$
(c, \boldsymbol{e})\left(c^{\prime}, \boldsymbol{e}^{\prime}\right)=\left(c c^{\prime}, c \boldsymbol{e}^{\prime}+\boldsymbol{e} c^{\prime}\right),
$$

where the product $c c^{\prime}$ is the ordinary product inside $C$, while $c \boldsymbol{e}^{\prime}$ and $\boldsymbol{e}^{\prime} c$ are defined as follows. Viewing $c \in C$ as an element of End $C_{C} \cong C$, then $\boldsymbol{e}_{1}=c \boldsymbol{e}^{\prime}$ is represented by the sequence obtained by pulling down the sequence $\boldsymbol{e}^{\prime}$ :

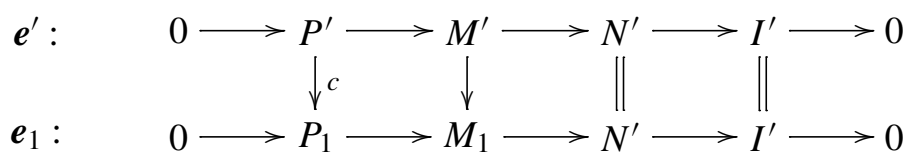

Similarly, if we view $c^{\prime} \in C$ as an element of End $D C_{C} \cong C$, then $\boldsymbol{e}_{2}=\boldsymbol{e} c^{\prime}$ is represented by the sequence obtained by lifting the sequence $\boldsymbol{e}$ : 


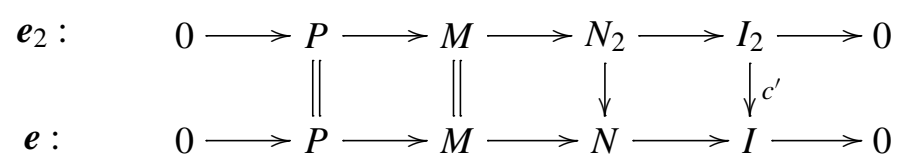

The following theorem allows us to view cluster-tilted algebras as relationextensions of tilted algebras.

Theorem 3.1 [Assem et al. 2008a]. An algebra $\Lambda$ is cluster-tilted of type $\Delta$ if and only if there exists a tilted algebra $C$ of type $\Delta$ such that $\Lambda$ is isomorphic to the relation-extension $\tilde{C}$ of $C$.

Every cluster-tilted algebra satisfies conditions (2) and (3) from Theorem 2.7 [Keller and Reiten 2007]. The bound quivers of cluster-tilted algebras of type $\mathbb{A}$ are explicitly described in [Buan and Vatne 2008, Proposition 3.1]. In fact they were already described in [Caldero et al. 2006] as the algebras $A(\Gamma)$ arising from a triangulation of an unpunctured polygon. The following proposition is contained in [Buan and Vatne 2008], but it also follows from Theorem 3.3 below.

Proposition 3.2 [Buan and Vatne 2008, (3.1)]. An algebra A is cluster-tilted of type $\mathbb{A}$ precisely when $A$ is gentle and there is a presentation $A=k Q / I$ which satisfies conditions (T1) and (T2) from Theorem 2.7.

In particular, the cluster-tilted algebras of type $\mathbb{A}$ are gentle. We describe in the following theorem, whose proof occupies the rest of the section, which of the gentle algebras are cluster-tilted:

Theorem 3.3. Let $C=k Q_{C} / I_{C}$ be a tilted algebra, and $\tilde{C}$ be its relation-extension. The following are equivalent.

(1) $C$ is gentle.

(2) $C$ is tilted of type $\mathbb{A}$ or $\tilde{A}$.

(3) $\tilde{C}$ is gentle.

(4) $\tilde{C}$ is cluster-tilted of type $\mathbb{A}$ or $\tilde{\mathbb{A}}$.

A preliminary part of the proof follows from a result in [Schröer 1999], which says that the class of gentle algebras is stable under tilting.

Lemma 3.4. If a tilted algebra is gentle, then it is tilted of type $\mathbb{A}$ or $\tilde{\mathbb{A}}$.

Proof. Let $\Delta$ be a quiver such that $C$ is tilted of type $\Delta$. Then there exists a tilting $C$-module $T$ such that End $T=k \Delta$. According to [Schröer 1999], $k \Delta$ is a gentle algebra. This implies that the quiver $\Delta$ is of type $\mathbb{A}$ or $\tilde{\mathbb{A}}$.

Lemma 3.5. If $\tilde{C}$ is gentle, then so is $C$.

Proof. This follows from the fact that $\tilde{C}$ is a split extension of $C$ and from [Assem et al. 2008b, (2.7)]. 
Lemma 3.6. The algebra $C$ is tilted of type $\mathbb{A}$ or $\tilde{\mathbb{A}}$ if and only if $\tilde{C}$ is cluster-tilted of type $\mathbb{A}$ or $\tilde{A}$.

Proof. Clearly, if $C$ is tilted of type $\mathbb{A}$ or $\tilde{A}$, then $\tilde{C}$ is cluster-tilted of type $\mathbb{A}$ or $\tilde{\mathbb{A}}$. Conversely, suppose $\tilde{C}$ is cluster-tilted of type $\mathbb{A}$ or $\tilde{\mathbb{A}}$. By [Assem et al. 2008a] there exists a local slice $\Sigma^{\prime}$ in $\bmod \tilde{C}$ such that $C^{\prime}=\tilde{C} /$ Ann $\Sigma^{\prime}$ is tilted of type $\mathbb{A}$ or $\tilde{\mathbb{A}}$. On the other hand, since $\tilde{C}=C \ltimes \operatorname{Ext}_{C}^{2}(D C, C)$, then there exists a local slice $\Sigma$ in $\bmod \tilde{C}$ such that $C=\tilde{C} / \operatorname{Ann} \Sigma$. Since both $\Sigma$ and $\Sigma^{\prime}$ have the same underlying graph, $C$ and $C^{\prime}$ have the same type, so $C$ is tilted of type $\mathbb{A}$ or $\tilde{\mathbb{A}}$.

The main part of the proof of Theorem 3.3 is concerned with the problem of showing that $\tilde{C}$ is gentle if $C$ is tilted of type $\mathbb{A}$ or $\tilde{A}$. This will be done next.

Relation-extensions of tilted algebras of types $\mathbb{A}$ and $\tilde{\mathbb{A}}$. Suppose $C=k Q_{C} / I_{C}$ is tilted of type $\mathbb{A}$ or $\tilde{\mathbb{A}}$. In particular, $C$ is gentle because of [Assem 1982] and [Assem and Skowroński 1987]. Moreover, the quiver of $\tilde{C}$ is known, as are some of its relations, namely those already in $C$ [Assem et al. 2008a; Assem et al. 2008b]. The aim here is to study the remaining relations of $\tilde{C}$.

First, the bound quiver of a tilted algebra of type $\mathbb{A}$ has been described in [Assem 1982], and that of a tilted algebra of type $\tilde{A}$ in [Roldán 1983]. The criterion given here is derived from [Huard and Liu 2000].

We recall that a double-zero in a gentle algebra is a reduced walk of the form $\alpha \beta \omega \gamma \delta$, where $\alpha, \beta, \gamma$ and $\delta$ are arrows such that $\alpha \beta$ and $\gamma \delta$ are relations, while $\omega$ is a nonzero reduced walk (that is, a walk which does not contain any relation). Note that $\omega$ may be trivial and that in this case $\beta$ and $\gamma$ may coincide.

Example. The algebra

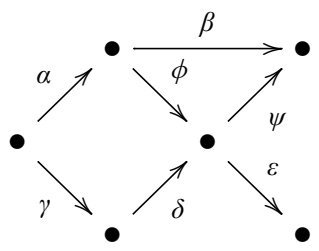

where $\alpha \beta=\phi \psi=\delta \varepsilon=0$, is gentle with a double-zero (namely $\phi \psi \beta^{-1} \phi \psi$ ).

Proposition 3.7 [Assem 1982; Assem and Skowroński 1987]. (1) An algebra is tilted of type $\mathbb{A}$ if and only if it admits a bound quiver presentation $k Q / I$, with $(Q, I)$ a gentle tree with no double-zero.

(2) An algebra is tilted of type $\tilde{A}$ if and only if it admits a bound quiver presentation $k Q / I$, with $(Q, I)$ a gentle presentation with no double-zero and a unique (nonoriented) cycle such that, if the cycle is a band, then all arrows attached to the cycle either enter it or leave it. 
Example. Consider the algebras given by the bound quivers
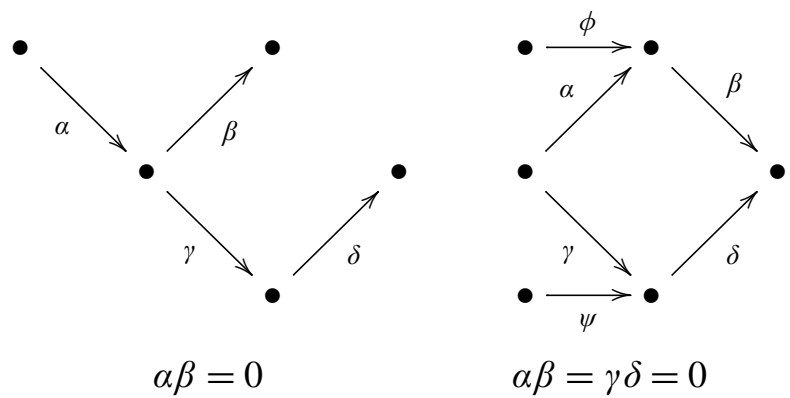

$$
\alpha \beta=0 \quad \alpha \beta=\gamma \delta=0
$$

Using Proposition 3.7, we see that the first one is tilted of type $\mathbb{A}$, while the second one is tilted of type $\tilde{\mathbb{A}}$.

A vanishing criterion. We need a criterion to verify whether a given exact sequence represents the zero element in the second extension group:

Lemma 3.8 [Happel et al. 1996, (II.1.3)]. Given a morphism $f: M \longrightarrow N$, the exact sequence

$$
0 \longrightarrow \operatorname{Ker} f \longrightarrow M \stackrel{f}{\longrightarrow} N \longrightarrow \text { Coker } f \longrightarrow 0
$$

represents the zero element of $\operatorname{Ext}^{2}(\operatorname{Coker} f, \operatorname{Ker} f)$ if and only if there exist a module $X$ and morphisms $g$, $h$ such that the sequence

$$
0 \longrightarrow M \stackrel{(p, g)^{t}}{\longrightarrow} \operatorname{Im} f \oplus X \stackrel{(j, h)}{\longrightarrow} N \longrightarrow 0
$$

is exact, where $p$ and $j$ are the natural morphisms arising from $f$.

The following lemma will be used frequently.

Lemma 3.9. Let $(Q, I)$ be a gentle presentation of an algebra $C$, and let $\alpha: c \longrightarrow b$ and $\beta: b \longrightarrow a$ be arrows in $Q$. Let $\sigma$ and $\eta$ be strings, not passing through $b$, such that $\beta \sigma$ and $\eta \alpha$ are strings. Let $f: M(\beta \sigma) \longrightarrow M(\eta \alpha)$ be a morphism such that $\operatorname{Im} f=S_{b}$.

Then the exact sequence

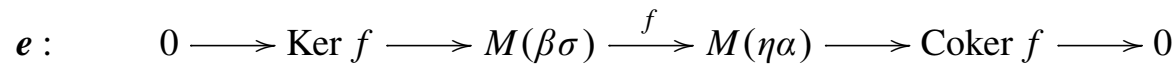

represents a nonzero element of $\operatorname{Ext}_{C}^{2}(\operatorname{Coker} f, \operatorname{Ker} f)$ if and only if $\alpha \beta$ lies in I.

Proof. In view of Lemma 3.8, the sequence $\boldsymbol{e}$ represents a nonzero element of $\operatorname{Ext}_{C}^{2}(\operatorname{Coker} f, \operatorname{Ker} f)$ if and only if there exists no short exact sequence of the form

$$
0 \longrightarrow M(\beta \sigma) \stackrel{(p, g)^{t}}{\longrightarrow} \operatorname{Im} f \oplus X \stackrel{(j, h)}{\longrightarrow} M(\eta \alpha) \longrightarrow 0,
$$


Assume such a sequence exists. Since $S_{b}$ appears exactly once as a composition factor of $M(\beta \sigma)$ and $M(\eta \alpha)$, then it also appears exactly once as a composition factor of $X$. Therefore, there exists a unique indecomposable summand $Y$ of $X$ admitting $S_{b}$ as a composition factor.

We claim that $g: M(\beta \sigma) \longrightarrow X$ is a monomorphism: let $x \in Q_{0}$ and take a vector $v \in M(\beta \sigma)_{x}$ such that $g_{x}(v)=0$. If $x \neq b$, then $p_{x}(v)=0$ and so $(p, g)_{x}^{t}(v)=0$ which implies $v=0$. If $x=b$, then $\left(p_{a}(\beta v), g_{a}\left(M(\beta \sigma)_{\beta}(v)\right)\right)^{t}=$ $(p, g)_{a}^{t}\left(M(\beta \sigma)_{\beta}(v)\right)=(\operatorname{Im} f \oplus X)_{\beta}(p, g)_{b}^{t}(v)=0$ which implies $M(\beta \sigma)_{\beta}(v)=0$. Since $\left(M(\beta \sigma)_{\beta}\right.$ is injective, then $v=0$. This completes the proof of our claim.

Since the evaluation $M(\beta \sigma)_{\beta}$ of the module $M(\beta \sigma)$ on the arrow $\beta$ is nonzero, we must have $X_{\beta} \neq 0$. Now, $S_{b}$ is a composition factor of $Y$, hence $Y_{\beta} \neq 0$ as well. Similarly, $h$ is an epimorphism and it follows that $Y_{\alpha} \neq 0$. On the other hand, $Y$ must be a string or a band module. The above reasoning implies that $\alpha \beta$ must then be a subpath of a string or a band, which implies that $\alpha \beta \notin I$, as required.

Conversely, if $\alpha \beta \notin I$, then we have a short exact sequence

$$
0 \longrightarrow M(\beta \sigma) \longrightarrow S_{b} \oplus M(\eta \alpha \beta \sigma) \longrightarrow M(\eta \alpha) \longrightarrow 0
$$

and hence $\boldsymbol{e}$ represents the zero element in $\operatorname{Ext}_{C}^{2}(\operatorname{Coker} f, \operatorname{Ker} f)$.

Arrows. From now on, let $C$ be a tilted algebra of type $\mathbb{A}$ or $\tilde{A}$. We give a description of the elements of $\tilde{C}=C \ltimes \operatorname{Ext}_{C}^{2}(D C, C)$ corresponding to the arrows of its ordinary quiver. In [Assem et al. 2008a, (2.4)], it is proved that the quiver of $\tilde{C}$ is obtained from that of $C$ by adding an arrow from $x$ to $y$ for each relation from $y$ to $x$. The elements of $\tilde{C}$ corresponding to the arrows of $C$ are of the form $(\alpha, 0)$, where $\alpha$ is an arrow of $C$.

The other arrows correspond to relations in $C$. Let $\alpha \beta$ be a relation from $c$ to $a$ in $C$, and let $\xi_{\alpha \beta}$ be the corresponding new arrow in $\tilde{C}$.

Lemma 3.10. The new arrow $\xi_{\alpha \beta}$ lies in $0 \oplus \operatorname{Ext}_{C}^{2}\left(I_{c}, P_{a}\right)$.

Proof. This new arrow lies in $e_{a} \tilde{C} e_{c}$, which can be written as the direct sum of $e_{a} C e_{c}$ and $e_{a} \operatorname{Ext}_{C}^{2}(D C, C) e_{c}$. We know from Proposition 3.7 that the quiver of $C$ contains no double-zero. Consequently, there are no paths from $a$ to $c$, and hence $e_{a} C e_{c}=0$. Moreover, $e_{a} \operatorname{Ext}_{C}^{2}(D C, C) e_{c}=\operatorname{Ext}_{C}^{2}\left(I_{c}, P_{a}\right)$. The element $\xi_{\alpha \beta}$ thus lies in $0 \oplus \operatorname{Ext}_{C}^{2}\left(I_{c}, P_{a}\right)$.

The following lemma gives the dimension and a basis of the extension space involved in the last expression.

Lemma 3.11. Let $\alpha: c \longrightarrow b$ and $\beta: b \longrightarrow a$ be two arrows of $C$ such that $\alpha \beta \in I_{C}$. 
(a) The dimension of the vector space $\operatorname{Ext}_{C}^{2}\left(I_{c}, P_{a}\right)$ is 1 or 2 . Its dimension is 2 if and only if the following situation occurs in the bound quiver of $C$ :

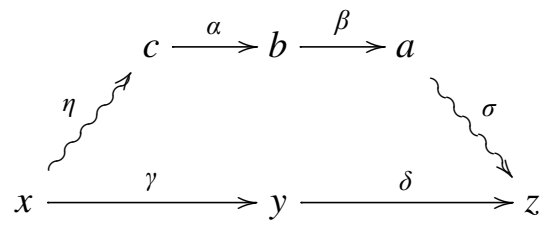

where $\gamma$ and $\delta$ are arrows, $\eta$ and $\sigma$ are paths, possibly stationary, without relations, and $\alpha \beta, \gamma \delta$ are relations.

(b) If the dimension of the space is 1 , then a basis is given by the sequence

$$
\boldsymbol{e}_{1}: \quad 0 \longrightarrow P_{a} \longrightarrow M(\beta \sigma) \longrightarrow M(\eta \alpha) \longrightarrow I_{c} \longrightarrow 0,
$$

where $\eta$ and $\sigma$ are paths such that $I_{c}=M(\eta)$ and $P_{a}=M(\sigma)$.

(c) If the dimension of the space is 2 , then a basis is given by the sequences

$$
\begin{array}{ll}
\boldsymbol{e}_{1}: & 0 \longrightarrow P_{a} \longrightarrow M(\beta \sigma) \longrightarrow M(\eta \alpha) \longrightarrow I_{c} \longrightarrow 0, \\
\boldsymbol{e}_{2}: & \left.0 \longrightarrow P_{a} \longrightarrow M\left(\sigma \delta^{-1}\right) \longrightarrow \gamma^{-1} \eta\right) \longrightarrow I_{c} \longrightarrow 0,
\end{array}
$$

where $\gamma, \delta, \eta$ and $\sigma$ are as in the figure in part (a).

Proof. (a) It is known from [Assem et al. 2008a] that there is a new arrow from $a$ to $c$; thus the dimension cannot be zero. On the other hand, since $C$ is gentle and without double-zero, the local situation of the relation $\alpha \beta$ can be described by the following figure, where dotted lines represent relations.

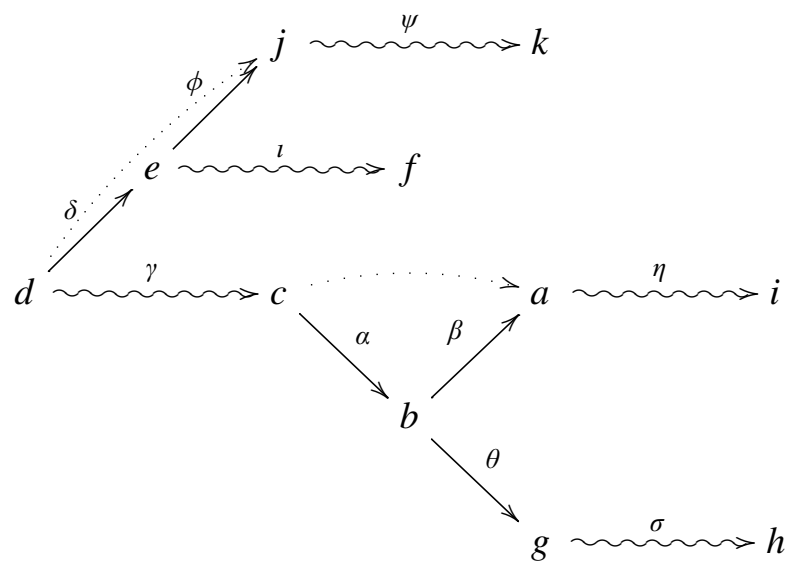

This diagram allows us to compute a projective resolution of $I_{C}$ in $\bmod C$ :

$$
0 \stackrel{p_{3}}{\longrightarrow} P(2) \stackrel{p_{2}}{\longrightarrow} P(1) \stackrel{p_{1}}{\longrightarrow} P(0) \stackrel{p_{0}}{\longrightarrow} I_{c} \longrightarrow 0,
$$


where $P(2)=M(\psi) \oplus M(\eta), P(1)=M\left(l^{-1} \phi \psi\right) \oplus M\left(\sigma^{-1} \theta^{-1} \beta \eta\right)$ and $P(0)=$ $M\left(l^{-1} \delta^{-1} \gamma \alpha \theta \sigma\right)$. Note that some direct summands of the terms of this sequence can be zero. Applying $\operatorname{Hom}_{C}\left(-, P_{a}\right)$, we get a complex

$$
\begin{aligned}
0 \longrightarrow \operatorname{Hom}_{C}\left(I_{c}, P_{a}\right) & \stackrel{\left(p_{0}, P_{a}\right)}{\longrightarrow} \operatorname{Hom}_{C}\left(P(0), P_{a}\right) \\
& \stackrel{\left(p_{1}, P_{a}\right)}{\longrightarrow} \operatorname{Hom}_{C}\left(P(1), P_{a}\right) \stackrel{\left(p_{2}, P_{a}\right)}{\longrightarrow} \operatorname{Hom}_{C}\left(P(2), P_{a}\right) \stackrel{\left(p_{3}, P_{a}\right)}{\longrightarrow} 0 .
\end{aligned}
$$

This yields

$$
\operatorname{Ext}_{C}^{2}\left(I_{c}, P_{a}\right)=\frac{\operatorname{Ker} \operatorname{Hom}\left(p_{3}, P_{a}\right)}{\operatorname{Im} \operatorname{Hom}\left(p_{2}, P_{a}\right)}=\frac{\operatorname{Hom}\left(M(\psi), P_{a}\right) \oplus \operatorname{Hom}\left(M(\eta), P_{a}\right)}{\operatorname{Im} \operatorname{Hom}\left(p_{2}, P_{a}\right)} .
$$

Since $P_{a}=M(\eta)$, we have $\operatorname{dim} \operatorname{Hom}\left(M(\eta), P_{a}\right)=1$, and since

$$
\operatorname{Hom}\left(M\left(\sigma^{-1} \theta^{-1} \beta \eta\right), P_{a}\right)=0,
$$

no nonzero morphism in $\operatorname{Hom}\left(M(\eta), P_{a}\right)$ factors through $p_{2}$.

We claim that $\operatorname{Hom}\left(M(\psi), P_{a}\right)$ is nonzero if and only if $j=i$. Indeed, a nonzero morphism from $M(\psi)$ to $P_{a}$ can only exist when $j$ coincides with a vertex on the path $\eta$. But if $j$ were a vertex different from $i$, then there would be an arrow $\phi^{\prime}: j \rightarrow j^{\prime}$ in the path $\eta$, forcing the relation $\phi \phi^{\prime \prime}$ and creating the double-zero $\delta \phi \phi^{\prime}$. Thus $j=i$. In this case, $\psi$ has no choice but to be the trivial path in $i$, and $\operatorname{dim} \operatorname{Hom}\left(M(\psi), P_{a}\right)=1$. Since $\operatorname{Hom}\left(M\left(l^{-1} \phi \psi, P_{a}\right)=0\right.$, no nonzero morphism in $\operatorname{Hom}\left(M(\psi), P_{a}\right)$ factors through $p_{2}$.

Hence no nonzero morphism in $\operatorname{Hom}\left(M(\psi), P_{a}\right) \oplus \operatorname{Hom}\left(M(\eta), P_{a}\right)$ factors through $p_{2}$. Thus the dimension of this space is either 1 or 2 , and it is 2 exactly when $i=j$. In this case, and in this case only, we have

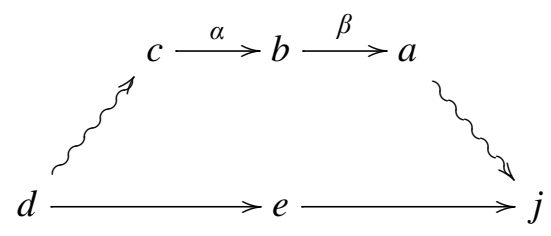

as desired.

(b) It follows from Lemma 3.9 that $\boldsymbol{e}_{1}$ is nonzero. The result follows.

(c) It follows from Lemma 3.9 that $\boldsymbol{e}_{1}$ and $\boldsymbol{e}_{2}$ are nonzero. It remains to be shown that $\boldsymbol{e}_{1}$ and $\boldsymbol{e}_{2}$ are linearly independent. Suppose there exists a nonzero scalar $\lambda$ such that $\boldsymbol{e}_{2}+\lambda \boldsymbol{e}_{1}=0$. Computing this sum, we get the sequence

$$
0 \longrightarrow P_{a} \longrightarrow M\left(\beta \sigma \delta^{-1}\right) \stackrel{f}{\longrightarrow} M\left(\gamma^{-1} \eta \alpha\right) \longrightarrow I_{c} \longrightarrow 0,
$$

where all morphisms are multiples of the natural morphisms between string modules. 
Here, applying Lemma 3.9 is not possible, since $\operatorname{Im} f=S_{b} \oplus S_{y}$, but a similar technique of proof can be used.

Suppose there exist a module $X$ and morphisms $g$ and $h$ such that the sequence

$$
0 \longrightarrow M\left(\beta \sigma \delta^{-1}\right) \stackrel{(p, g)^{t}}{\longrightarrow}\left(S_{b} \oplus S_{y}\right) \oplus X \stackrel{(j, h)}{\longrightarrow} M\left(\gamma^{-1} \eta \alpha\right) \longrightarrow 0
$$

is exact, where $f=j p$ is the canonical factorisation. Since $S_{b}$ appears exactly once as a composition factor of $M\left(\beta \sigma \delta^{-1}\right)$ and $M\left(\gamma^{-1} \eta \alpha\right)$, it also appears exactly once as a composition factor of $X$. Therefore, there exists a unique indecomposable summand $Y$ of $X$ admitting $S_{b}$ as a composition factor. As in the proof of Lemma 3.9, we show that $Y_{\beta} \neq 0$ and $Y_{\alpha} \neq 0$. Therefore, $\alpha \beta$ must be a subpath of a string or a band, which is a contradiction, since it is a relation.

The sequences $\boldsymbol{e}_{1}$ and $\boldsymbol{e}_{2}$ thus form a basis of the extension space.

It remains to determine which of the basis elements are represented by arrows of $\tilde{C}$.

Lemma 3.12. Let $\alpha: c \longrightarrow b$ and $\beta: b \longrightarrow a$ be two arrows of the quiver of $C$ such that $\alpha \beta$ is a relation. Let $\xi_{\alpha \beta}$ be the corresponding new arrow in $\tilde{C}$. With the notation of Lemma 3.11, the element $\left(0, \boldsymbol{e}_{1}\right)$ can be chosen to represent $\xi_{\alpha \beta}$.

Proof. The space $0 \oplus \operatorname{Ext}_{C}^{2}\left(I_{c}, P_{a}\right)$ contains at least one arrow.

If its dimension is 1 , the result is obvious.

If its dimension is 2, Lemma 3.11 describes the situation of $\alpha \beta$ in the quiver of $C$. Two cases arise.

First, suppose that $\eta$ and $\sigma$ are both trivial paths.

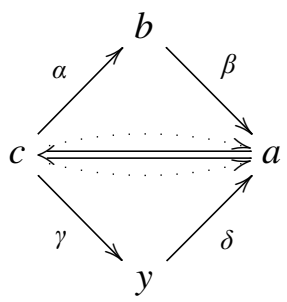

In this case, two arrows from $a$ to $c$ are added to the quiver. Both $\left(0, \boldsymbol{e}_{1}\right)$ and $\left(0, \boldsymbol{e}_{2}\right)$ must thus represent arrows of $\tilde{C}$.

Second, suppose $\eta$ and $\sigma$ are not both trivial. In this case, Lemma 3.11 implies that $\operatorname{Ext}_{C}^{2}\left(I_{x}, P_{z}\right)$ is of dimension 1, and that a basis is given by

$$
\boldsymbol{e}^{\prime}: \quad 0 \longrightarrow P_{z} \longrightarrow M(\delta) \longrightarrow M(\gamma) \longrightarrow I_{x} \longrightarrow 0 .
$$

Reasoning as above, we get that $\left(0, \boldsymbol{e}^{\prime}\right)$ represents the new arrow from $z$ to $x$. Moreover, a straightforward calculation yields $(\sigma, 0)\left(0, \boldsymbol{e}^{\prime}\right)(\eta, 0)=\left(0, \boldsymbol{e}_{2}\right)$. 
Since one of $\eta$ and $\sigma$ is not trivial, one of $(0, \eta)$ and $(0, \sigma)$ must lie in $\operatorname{rad} \tilde{C}$. Therefore $\left(0, \boldsymbol{e}_{2}\right) \in \operatorname{rad}^{2} \tilde{C}$, and $\left(0, \boldsymbol{e}_{1}\right) \in \operatorname{rad} \tilde{C} \backslash \operatorname{rad}^{2} \tilde{C}$; in other words, $\left(0, \boldsymbol{e}_{1}\right)$ represents an arrow from $a$ to $c$.

Relations. Knowing how to write arrows in $\tilde{C}$ allows us to compute the relations.

Lemma 3.13. Let $C=k Q_{C} / I_{C}$ and $\tilde{C}=k Q_{\tilde{C}} / I_{\tilde{C}}$.

(1) Let $\omega_{1}, \omega_{2}, \ldots, \omega_{n}$ be paths from $x$ to $y$ in the quiver of $C$, and let $\lambda_{1}, \lambda_{2}, \ldots$, $\lambda_{n} \in k$. Then $\sum_{i=1}^{n} \lambda_{i}\left(\omega_{i}, 0\right)=0$ in $\tilde{C}$ if and only if $\sum_{i=1}^{n} \lambda_{i} \omega_{i}=0$ in $C$.

(2) Let $\alpha: c \longrightarrow b$ and $\beta: b \longrightarrow a$ be two arrows in the quiver of $C$ such that $\alpha \beta$ is a relation. Let $\left(0, \boldsymbol{e}_{1}\right)$ be the element representing the corresponding new arrow, where $\boldsymbol{e}_{1}$ is as in Lemma 3.11. Then $\left(0, \boldsymbol{e}_{1}\right)(\alpha, 0)=0$ and $(\beta, 0)\left(0, \boldsymbol{e}_{1}\right)=0$.

(3) The ideal $I_{\tilde{C}}$ is generated by the relations of $C$ and those described in (2).

Proof. (1) This is shown in [Assem et al. 2008b].

(2) Viewing $\alpha$ as an element of End $D C$, or more precisely as a morphism from $I_{b}$ to $I_{c}$, we can compute $\boldsymbol{e}_{1} \beta$ :

$$
\boldsymbol{e}_{1} \beta: \quad 0 \longrightarrow P_{a} \longrightarrow M(\beta \sigma) \longrightarrow M(\eta \alpha) \oplus M(\varphi \gamma) \longrightarrow I_{b} \longrightarrow 0,
$$

where $I_{b}=M\left(\eta \alpha \gamma^{-1} \varphi^{-1}\right)$. This sequence represents the zero element, because of Lemma 3.8 and exactness of the sequence

$$
0 \longrightarrow M(\beta \sigma) \longrightarrow S_{b} \oplus M(\varphi \gamma \beta \sigma) \oplus M(\eta \alpha) \longrightarrow M(\varphi \gamma) \oplus M(\eta \alpha) \longrightarrow 0 .
$$

Therefore $\left(0, \boldsymbol{e}_{1}\right)(\alpha, 0)=0$.

In a dual way, we prove that $(\beta, 0)\left(0, \boldsymbol{e}_{1}\right)=0$.

(3) It is sufficient to show that new arrows in the quiver of $\tilde{C}$ are not involved in other relations than those described in (2).

First suppose that $w$ is a monomial relation involving new arrows and relations other than those described in (2). Then it must contain exactly one new arrow $\xi$, corresponding to a relation $\alpha \beta$; otherwise the quiver of $C$ would contain a double-zero. Write $w=u \xi v$, where $u$ and $v$ are nonzero paths consisting of arrows of $C$. Let $\boldsymbol{e}_{1}$ be the sequence as in Lemma 3.11 corresponding to $\xi$. Then $(u, 0)\left(0, \boldsymbol{e}_{1}\right)(v, 0)=\left(0, u \boldsymbol{e}_{1} v\right)$, where $u \boldsymbol{e}_{1} v$ is the sequence

$$
0 \longrightarrow M\left(u^{-1} u^{\prime}\right) \longrightarrow M\left(\beta u^{-1} u^{\prime}\right) \longrightarrow M\left(v^{\prime} v^{-1} \alpha\right) \longrightarrow M\left(v^{\prime} v^{-1}\right) \longrightarrow 0,
$$

where $u^{\prime}$ and $v^{\prime}$ are paths in the quiver of $C$. The figure at the top of the next page illustrates the local situation, where $\alpha \beta=\gamma^{\prime} \delta^{\prime}=0$; the last arrow of $u$ and the first 
of $t$ form a relation, as do the last of $v$ and $v^{\prime}$ and the first of $w^{\prime}$ and $w$, respectively.

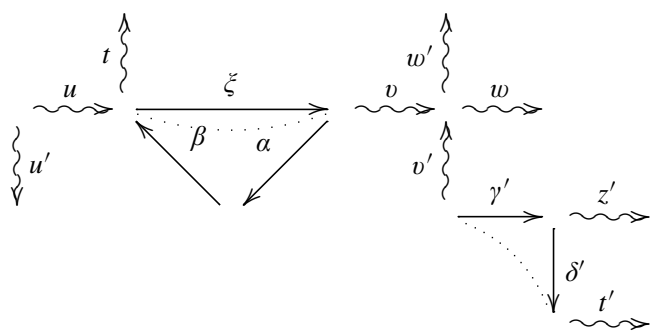

This yields the following commutative diagram, where the first line is a projective resolution of $M\left(v^{\prime} v^{-1}\right)$ :

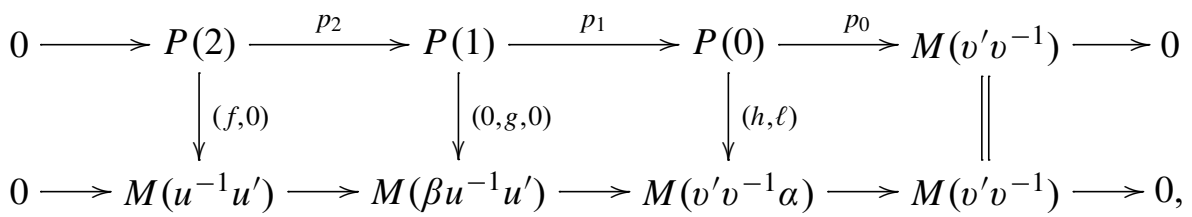

where $P(2)=M(t) \oplus M\left(t^{\prime}\right), P(1)=M\left(w^{\prime} w\right) \oplus M\left(z^{-1} \gamma^{-1} \beta t\right) \oplus M\left(z^{\prime-1} \delta^{\prime} t^{\prime}\right)$ and $P(0)=M\left(z^{-1} \gamma^{-1} \alpha^{-1} v w\right) \oplus M\left(z^{\prime-1} \gamma^{\prime-1} v^{\prime} w^{\prime}\right)$, and all nonzero morphisms are the natural morphisms between string modules. It is then seen that $(f, 0)$ cannot factor through $p_{2}$, and thus the lower exact sequence is nonzero. Hence there are no other monomial relations than those in (2).

Now suppose we have a minimal relation of the form $\sum_{\tilde{C}}^{m} \lambda_{i} w_{i}$, where each $\lambda_{i}$ is a nonzero scalar, each $w_{i}$ is a path in the quiver of $\tilde{C}$, and $m \geq 2$. At least one of the $w_{i}$ must pass through a new arrow, and since $C$ contains no double zero, this implies that each $w_{i}$ must pass through exactly one new arrow, say $\xi_{i}$, corresponding to a relation $\alpha_{i} \beta_{i}$. Write $w_{i}=u_{i} \xi_{i} v_{i}$, where $u_{i}$ and $v_{i}$ are paths of the quiver of $C$.

Since the quiver of $C$ contains at most one cycle, we must have $m=2$. Since $k$ is a field, we may suppose that $\lambda_{1}=1$. Letting $\boldsymbol{e}_{1}$ and $\boldsymbol{e}_{2}$ be the sequences associated to $\xi_{1}$ and $\xi_{2}$, respectively, we get that $u_{1} \boldsymbol{e}_{1} v_{1}$ and $\lambda_{2} u_{2} \boldsymbol{e}_{2} v_{2}$ are both sequences of the form above. Their sum is the sequence

$$
0 \longrightarrow M\left(u_{2}^{-1} u_{1}\right) \longrightarrow M\left(\beta_{2} u_{2}^{-1} u_{1} \beta_{1}^{-1}\right)
$$

$$
\longrightarrow M\left(\alpha_{2}^{-1} v_{2} v_{1}^{-1} \alpha_{1}\right) \longrightarrow M\left(v_{1} v_{2}^{-1}\right) \longrightarrow 0 .
$$

By an argument similar to the one given in the proof of Lemma 3.11(c), this element is not zero; a contradiction. Hence no binomial relations exist in $\tilde{C}$.

The relations described in the preceding lemma make $\tilde{C}$ a gentle algebra.

Lemma 3.14. If $\tilde{C}$ is cluster-tilted of type $\mathbb{A}$ or $\tilde{A}$, then $\tilde{C}$ is gentle. 
Proof. The relations of $\tilde{C}$ are known (see Lemma 3.13). Moreover, $C$ is gentle.

Suppose that there are $r$ new arrows. Let us add the new arrows and the corresponding new relations one by one, thus obtaining a sequence $C=C_{0}, C_{1}, \ldots$, $C_{r}=\tilde{C}$ of algebras. We show that $C_{i}$ is gentle for all $i$ in $\{0,1,2, \ldots, r\}$.

Since $C$ is gentle, then so is $C_{0}$. Suppose that $C_{i}$ is gentle, where $i$ is in $\{0,1,2, \ldots, r-1\}$. To get $C_{i+1}$, we add one new arrow, say $\gamma$ from $x$ to $y$. This arrow comes from a relation $\alpha \beta$ from $y$ to $x$ in $C$. We must add the relations $\beta \gamma$ and $\gamma \alpha$ to obtain $C_{i+1}$.

Since $C_{i}$ is gentle, there were already at most two arrows starting from $x$ in $C_{i}$. Suppose that there were two, say $\eta_{1}$ and $\eta_{2}$. Since $C_{i}$ is gentle, then $\beta$ is involved in a relation with one of the two, say $\eta_{1}$. The arrow $\eta_{1}$ cannot be in $C$, otherwise there would be a double zero involving $\alpha \beta$ and $\beta \eta_{1}$. So the arrow $\eta_{1}$ comes from a relation $\sigma \beta$ in $C$. Since $C$ is gentle, we must have that $\sigma=\alpha$, so that $\eta_{1}=\gamma$, which is absurd because $\gamma$ is not in $C_{i}$.

Therefore, in $C_{i}$, there is at most one outgoing arrow from $x$, and this arrow is not involved in a relation with $\beta$. This shows that in $C_{i+1}$, there are at most two arrows starting from $x$, say $\eta$ and $\gamma$, and that $\beta \eta$ is not a relation while $\beta \gamma$ is. Moreover, there is at most one more arrow ending in $x$, say $\delta$, and since $C_{i}$ is gentle, we have that $\delta \eta$ is a relation, while $\delta \gamma$ is not. So the relations at $x$ are those found in a gentle algebra.

Using a similar argument for the vertex $y$, we get that $C_{i+1}$ is a gentle algebra. By induction, $\tilde{C}$ is a gentle algebra.

Example. Lemma 3.13 allows us to compute the relation-extension of any gentle tilted algebra. As an illustration, consider the two algebras given in the example on page 216. The relation-extension of each is given in the following diagram:
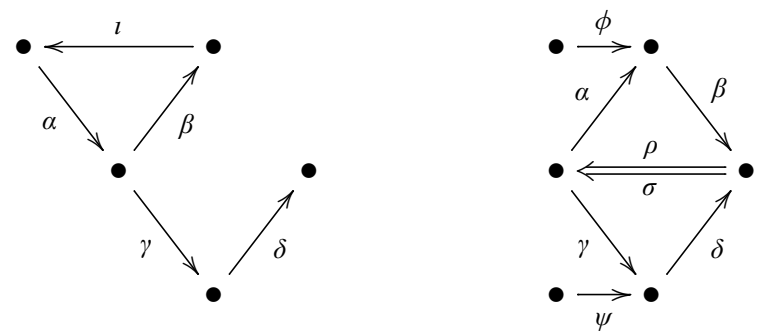

$$
\begin{array}{r}
\alpha \beta=\imath \alpha=\beta \imath=0 \quad \gamma \delta=\rho \alpha=0 \\
\beta \rho=\sigma \gamma=\delta \sigma=0
\end{array}
$$

Proof of the main theorem. Now the proof of Theorem 3.3, developed in separate parts over the last several pages, can be stated properly. 
Proof of Theorem 3.3. That (1) implies (2) is shown in Lemma 3.4, that (2) implies (4) in Lemma 3.6, that (4) implies (3) in Lemma 3.14, and that (3) implies (1) in Lemma 3.5.

\section{Geometry of surfaces and $A(\Gamma)$}

We study in this section more connections between geometric properties of the marked surface $(S, M)$ and properties of the algebra $A(\Gamma)$ given by a triangulation of $(S, M)$.

Cluster-tilted algebras arising from surfaces. We first address the question of which of the algebras $A(\Gamma)$ are cluster-tilted. Recall that all algebras $A(\Gamma)$ share the properties (2) and (3) from Theorem 2.7 with every cluster-tilted algebra. Moreover, it is shown in [Caldero et al. 2006] and [Buan and Vatne 2008] that the cluster-tilted algebras of type $\mathbb{A}$ are algebras $A(\Gamma)$ arising from a triangulation of an unpunctured polygon. In this section, we show the following generalization:

Theorem 4.1. Let $A(\Gamma)$ be the algebra associated to the triangulation $\Gamma$ of an unpunctured marked surface $(S, M)$. Then the following statements are equivalent:

(1) The algebra $A(\Gamma)$ is cluster-tilted.

(2) The algebra $A(\Gamma)$ is cluster-tilted of type $\mathbb{A}$ or $\tilde{\mathbb{A}}$.

(3) $S$ is a disc or an annulus.

Moreover, all cluster-tilted algebras of type $\mathbb{A}($ or $\tilde{\mathbb{A}})$ are of the form $A(\Gamma)$ for some triangulation $\Gamma$ of a disc $S$ (or an annulus $S$, respectively).

Proof. It is clear that (2) implies (1). Let us show the converse: Suppose that the algebra $A(\Gamma)$ is cluster-tilted. Thus there is a sequence of mutations transforming the quiver with potential defining $A(\Gamma)$ into some quiver $Q$ with zero potential. This sequence of mutations corresponds to a sequence of flips, transforming the triangulation $\Gamma$ of $(S, M)$ into a triangulation $T$ with $Q(T)=Q$ and zero potential. Hence $A(T)=k Q$ is hereditary. Since we know from Theorem 2.7 that $A(T)$ is gentle, this leaves only the possibilities that $Q$ is of type $\mathbb{A}$ or $\widetilde{\mathbb{A}}$. Therefore the algebra $A(\Gamma)$ is cluster-tilted of type $\mathbb{A}$ or $\tilde{A}$.

We prove now the equivalence of (2) and (3). Since all triangulations on $(S, M)$ are flip-equivalent [Hatcher 1991] and flips of the triangulation correspond to mutations of the corresponding quiver with potential [Labardini-Fragoso 2009], it is sufficient to consider one particular triangulation. In the case where $S$ is a disc, we choose the triangulation to be in the form of a fan, giving rise to a linear oriented quiver of type $\mathbb{A}$. In the case where $S$ is an annulus, we choose the triangulation given by two fans in opposite direction as shown in the figure at the top of the next page (where the left and right vertical edge should be identified). 


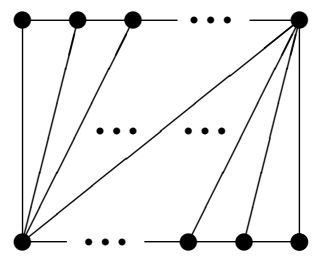

The corresponding quiver is of type $\tilde{\mathbb{A}}$ with zero potential; thus (3) implies (2). Conversely, we know from Proposition 2.8 that the quivers $Q(\Gamma)$ uniquely determine the topology of the unpunctured marked surface $(S, M)$. Therefore $S$ is a disc or an annulus, respectively, and since all triangulations are flip-equivalent, it is clear that all cluster-tilted algebras of the corresponding type occur.

Curves in ( $S, M)$ and string modules. In this section we are comparing strings in $A(\Gamma)$ to curves in $(S, M)$. By a curve in $(S, M)$ we mean a curve $\gamma$ in $S$ whose endpoints lie in $M$ and where all points except the endpoints lie in the interior of $S$. We usually consider curves up to homotopy. For instance, for two distinct curves $\gamma$ and $\delta$ in $(S, M)$, the intersection number $I_{\Gamma}(\gamma, \delta)$ is defined as the minimal number of transversal intersections of two representatives of the homotopy classes of $\gamma$ and $\delta$. Denote the internal arcs of the triangulation $\Gamma$ by $\left\{a_{1}, \ldots, a_{n}\right\}$. Then we define the intersection vector $I_{\Gamma}(\gamma)$ of a curve $\gamma$ as

$$
I_{\Gamma}(\gamma)=\left(I_{\Gamma}\left(\gamma, a_{1}\right) \ldots, I_{\Gamma}\left(\gamma, a_{n}\right)\right) .
$$

Proposition 4.2. Let $\Gamma$ be a triangulation of $(S, M)$, an unpunctured marked surface. Then there exists a bijection $\{\gamma\} \mapsto w(\gamma)$ between the homotopy classes of curves in $(S, M)$ not homotopic to an arc in $\Gamma$ and the strings of $A(\Gamma)$. Under this bijection, the intersection vector corresponds to the dimension vector of the corresponding string module, that is,

$$
I_{\Gamma}(\gamma)=\underline{\operatorname{dim}} M(w(\gamma)) .
$$

Proof. Let $w=x_{1} \stackrel{\alpha_{1}}{\longleftrightarrow} x_{2} \stackrel{\alpha_{2}}{\longleftrightarrow} \cdots \stackrel{\alpha_{s-1}}{\longleftrightarrow} x_{s}$ be a string in $A(\Gamma)$. We define a curve $\gamma(w)$ in $(S, M)$ as follows: The arcs $x_{1}$ and $x_{2}$ belong to the same triangle $T_{1}$ since they are joined by an arrow in $A(\Gamma)$. We connect the midpoints of $x_{1}$ and $x_{2}$ by a curve $\gamma_{1}$ in the interior of $T_{1}$. Proceeding in the same way with the remaining $\operatorname{arcs} x_{2}, \ldots, x_{s}$ we obtain curves $\gamma_{2}, \ldots, \gamma_{s-1}$ connecting the midpoints of the respective arcs. The internal arc $x_{1}$ belongs to two triangles: the triangle $T_{1}$ which we considered above and another triangle $T_{0}$. Let $P \in M$ be the marked point in $T_{0}$ opposite to the arc $x_{1}$. We now connect $P$ with the midpoint of $x_{1}$ by a curve $\gamma_{0}$ in the interior of $T_{0}$, and proceed in the same way on the other end of the string $w$, connecting the midpoint of $x_{s}$ with a marked point $Q$ by some curve $\gamma_{s}$. The curve $\gamma(w)$ is then defined as the concatenation of the curves $\gamma_{0}, \ldots, \gamma_{s}$. 

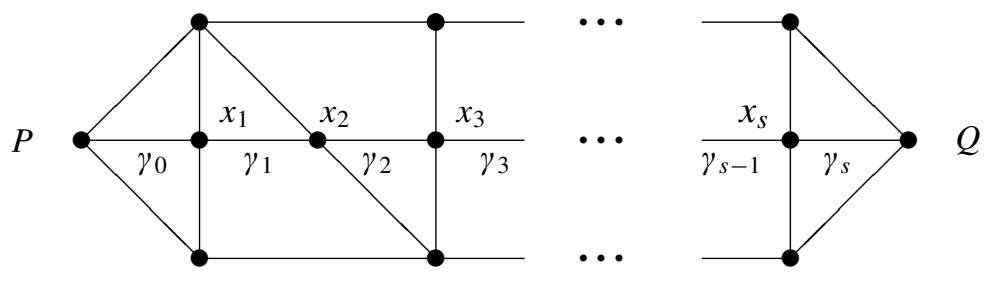

By construction, the points of intersection of the curve $\gamma(w)$ with arcs in $\Gamma$ are indexed by the vertices of the string $w$. The curve intersects the $\operatorname{arcs}$ of $\Gamma$ transversally, and since the string $w$ is reduced, none of the $\gamma_{i}$ is homotopic to a piece of an arc in $\Gamma$. Thus the intersection numbers are minimal, and $I_{\Gamma}(\gamma(w))=$ $\underline{\operatorname{dim}} M(w)$. Since $\gamma(w)$ has nontrivial intersection with arcs of $\Gamma$, it is clear that it is not homotopic to an arc in the triangulation $\Gamma$.

Conversely, let $\gamma:[0,1] \rightarrow S$ be a curve in $(S, M)$ which is not homotopic to an arc in $\Gamma$. We assume that the curve $\gamma$ is chosen (in its homotopy class) such that it intersects the arcs $a$ of $\Gamma$ transversally (if at all) and such that the intersection numbers $I_{\Gamma}(\gamma, a)$ are minimal. Orienting $\gamma$ from $P=\gamma(0) \in M$ to $Q=\gamma(1) \in M$, we denote by $x_{1}$ the first internal arc of $\Gamma$ that intersects $\gamma$, by $x_{2}$ the second arc, and so on. We thus obtain a sequence $x_{1}, \ldots, x_{s}$ of (not necessarily different) internal arcs in $\Gamma$. Since the intersection numbers are minimal, we know that $x_{i} \neq x_{i+1}$. Thus there are arrows, either $\alpha_{i}: x_{i} \rightarrow x_{i+1}$ or $\alpha_{i}: x_{i+1} \rightarrow x_{i}$ in $Q(\Gamma)$, and we obtain a walk $w(\gamma)=x_{1} \stackrel{\alpha_{1}}{\longleftrightarrow} x_{2} \stackrel{\alpha_{1}}{\longleftrightarrow} \cdots \stackrel{\alpha_{s-1}}{\longleftrightarrow} x_{s}$ in $Q(\Gamma)$. The fact that $\gamma$ intersects the arcs of $\Gamma$ transversally implies that the walk $w(\gamma)$ is reduced and avoids the zero-relations, and thus $w(\gamma)$ is a string in $A(\Gamma)$.

It follows from their construction that the two maps between strings and homotopy classes of curves defined above are mutually inverse.

Remark. Recall that two string modules $M(w)$ and $M(v)$ are isomorphic precisely when $v=w$ or $v=w^{-1}$. The inverse string $w^{-1}$ corresponds to orienting the curve in the opposite direction.

Proposition 4.3. Let $\Gamma$ be a triangulation of $(S, M)$, an unpunctured marked surface. Then there exists a bijection between the homotopy classes of closed curves in $(S, M)$ and powers $b^{n}$ of bands $b$ of $A(\Gamma)$.

The proof is analogous to that of the previous proposition.

An example where $A(\Gamma)$ is not cluster-tilted. We finally present in this section an example of an algebra $A(\Gamma)$ which is not cluster-tilted. Recall that an algebra $A$ is tame if for all $d \in \mathbb{N}$ there is a finite number $n_{d}$ of one-parameter families of $A$-modules such that almost every $d$-dimensional $A$-module belongs to one of these $n_{d}$ families. The algebra $A$ is said to be domestic if there is a constant $c$ such that $n_{d} \leq c$ for all $d \in \mathbb{N}$. On the other hand, if the numbers $n_{d}$ grow faster than any polynomial, then the tame algebra $A$ is said to be of nonpolynomial growth. 
It is well-known that every string algebra $A$ is tame, and that the one-parameter families are given by the bands in $A$ [Butler and Ringel 1987]. In particular, all the algebras $A(\Gamma)$ are tame, because they are gentle and thus string algebras. Moreover the tame cluster-tilted algebras of the form $A(\Gamma)$ studied here are all domestic; in fact, they are of type $\mathbb{A}$ or $\tilde{\mathbb{A}}$, and thus we may assume above that $c=0$ or $c=1$. We construct in this section an example of an algebra $A(\Gamma)$ which is of nonpolynomial growth, and thus cannot be cluster-tilted. To obtain this example, we consider a sphere $S$ with three holes and choose one marked point in each boundary component. We fix the following triangulation $\Gamma$ of $(S, M)$ :

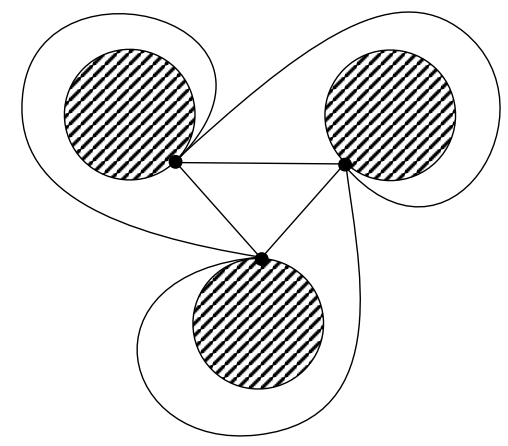

Then the algebra $A(\Gamma)$ is given by the following quiver with relations $\epsilon_{i} \rho_{i}=$ $0, \rho_{i} \sigma_{i}=0$ and $\sigma_{i} \epsilon_{i}=0$ for $i=1$ and $i=2$.

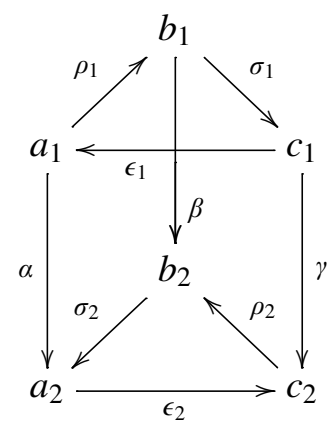

The string algebra $A(\Gamma)$ admits the two bands

$$
\begin{aligned}
& \xi=b_{2} \stackrel{\sigma_{2}}{\longrightarrow} a_{2} \stackrel{\alpha}{\longleftarrow} a_{1} \stackrel{\rho_{1}}{\longrightarrow} b_{1} \stackrel{\beta}{\longrightarrow} b_{2}, \\
& \eta=b_{2} \stackrel{\rho_{2}}{\longleftarrow} c_{2} \stackrel{\gamma}{\longleftarrow} c_{1} \stackrel{\sigma_{1}}{\longleftarrow} b_{1} \stackrel{\beta}{\longrightarrow} b_{2} .
\end{aligned}
$$

Since $\xi$ and $\eta$ can be composed arbitrarily, the number of bands of a fixed length $l$ grows exponentially with $l$, so the algebra $A(\Gamma)$ is of nonpolynomial growth.

We would like to point out that the notion of nonpolynomial growth of tame algebras discussed here does not coincide with the notion of nonpolynomial growth cluster algebras discussed in [Fomin et al. 2008]: There one counts the number of 
cluster variables, that is to say, of $\operatorname{arcs}$ in $(S, M)$, instead of one-parameter families, that is to say, closed curves in $(S, M)$. In [Fomin et al. 2008] the example we are considering in this section is classified as being of polynomial growth, meaning that, even if the number of curves is growing exponentially, the number of arcs is bounded for the sphere with three holes.

\section{References}

[Assem 1982] I. Assem, “Tilted algebras of type $\mathbf{A}_{n}$ ", Comm. Algebra 10:19 (1982), 2121-2139. MR 84c:16028 Zbl 0495.16028

[Assem and Skowroński 1987] I. Assem and A. Skowroński, "Iterated tilted algebras of type $\tilde{\mathbf{A}}_{n}$ ", Math. Z. 195:2 (1987), 269-290. MR 88m:16033 Zbl 0601.16022

[Assem et al. 2006] I. Assem, D. Simson, and A. Skowroński, Elements of the representation theory of associative algebras, Vol. 1: Techniques of representation theory, London Mathematical Society Student Texts 65, Cambridge University Press, 2006. MR 2006j:16020 Zbl 1092.16001

[Assem et al. 2008a] I. Assem, T. Brüstle, and R. Schiffler, "Cluster-tilted algebras as trivial extensions”, Bull. Lond. Math. Soc. 40:1 (2008), 151-162. MR 2009c:16086 Zbl 05267878

[Assem et al. 2008b] I. Assem, F. U. Coelho, and S. Trepode, "The bound quiver of a split extension”, J. Algebra Appl. 7:4 (2008), 405-423. MR 2009g:16022 Zbl 05381819

[Barot et al. 2008] M. Barot, E. Fernández, M. I. Platzeck, N. I. Pratti, and S. Trepode, "From iterated tilted algebras to cluster-tilted algebras", preprint, 2008. arXiv 0811.1328

[Brüstle 2001] T. Brüstle, "Kit algebras", J. Algebra 240:1 (2001), 1-24. MR 2002c:16021 Zbl 0991.16006

[Buan and Vatne 2008] A. B. Buan and D. F. Vatne, "Derived equivalence classification for clustertilted algebras of type $A_{n}$ ", J. Algebra 319:7 (2008), 2723-2738. MR 2009j:16013 Zbl 1155.16010

[Buan et al. 2006] A. B. Buan, R. Marsh, M. Reineke, I. Reiten, and G. Todorov, "Tilting theory and cluster combinatorics", Adv. Math. 204:2 (2006), 572-618. MR 2007f:16033 Zbl 1127.16011

[Buan et al. 2007] A. B. Buan, R. J. Marsh, and I. Reiten, "Cluster-tilted algebras", Trans. Amer. Math. Soc. 359:1 (2007), 323-332. MR 2007f:16035 Zbl 1123.16009

[Butler and Ringel 1987] M. C. R. Butler and C. M. Ringel, "Auslander-Reiten sequences with few middle terms and applications to string algebras", Comm. Algebra 15:1-2 (1987), 145-179. MR 88a:16055 Zbl 0612.16013

[Caldero et al. 2006] P. Caldero, F. Chapoton, and R. Schiffler, "Quivers with relations arising from clusters ( $A_{n}$ case)", Trans. Amer. Math. Soc. 358:3 (2006), 1347-1364. MR 2007a:16025 Zbl 1137.16020

[Derksen et al. 2008] H. Derksen, J. Weyman, and A. Zelevinsky, "Quivers with potentials and their representations, I: Mutations”, Selecta Math. (N.S.) 14:1 (2008), 59-119. MR 2480710 Zbl 05573998

[Fomin and Zelevinsky 2002] S. Fomin and A. Zelevinsky, "Cluster algebras, I: Foundations", J. Amer. Math. Soc. 15:2 (2002), 497-529. MR 2003f:16050 Zbl 1101.76415

[Fomin et al. 2008] S. Fomin, M. Shapiro, and D. Thurston, "Cluster algebras and triangulated surfaces, I: Cluster complexes”, Acta Math. 201:1 (2008), 83-146. MR 2448067 Zbl 05508850

[Gabriel 1981] P. Gabriel, "The universal cover of a representation-finite algebra”, pp. 68-105 in Representations of algebras (Puebla, 1980), edited by M. Auslander and E. Lluis, Lecture Notes in Math. 903, Springer, Berlin, 1981. MR 83f:16036 Zbl 0481.16008 
[Happel et al. 1996] D. Happel, I. Reiten, and S. O. Smalø, Tilting in abelian categories and quasitilted algebras, Mem. Amer. Math. Soc. 575, Amer. Math. Soc., Providence, 1996. MR 97j:16009 Zbl 0849.16011

[Hatcher 1991] A. Hatcher, “On triangulations of surfaces”, Topology Appl. 40:2 (1991), 189-194. MR 92f:57020 Zbl 0727.57012

[Huard and Liu 2000] F. Huard and S. Liu, "Tilted string algebras", J. Pure Appl. Algebra 153:2 (2000), 151-164. MR 2001j:16018 Zbl 0962.16009

[Keller 2007] B. Keller, "Triangulated Calabi-Yau categories", in Proceedings of the workshop of the ICRA 12 (Torun 2007), 2007.

[Keller 2009] B. Keller, "Deformed Calabi-Yau completions”, preprint, 2009. arXiv 0908.3499v5

[Keller and Reiten 2007] B. Keller and I. Reiten, "Cluster-tilted algebras are Gorenstein and stably Calabi-Yau”, Adv. Math. 211:1 (2007), 123-151. MR 2008b:18018 Zbl 1128.18007

[Labardini-Fragoso 2009] D. Labardini-Fragoso, "Quivers with potentials associated to triangulated surfaces”, Proc. Lond. Math. Soc. (3) 98:3 (2009), 797-839. MR 2500873 Zbl 05551833

[Massey 1991] W. S. Massey, A basic course in algebraic topology, Graduate Texts in Mathematics 127, Springer, New York, 1991. MR 92c:55001 Zbl 0725.55001

[Pogorzały and Skowroński 1991] Z. Pogorzały and A. Skowroński, "Self-injective biserial standard algebras”, J. Algebra 138:2 (1991), 491-504. MR 92f:16012 Zbl 0808.16019

[Roldán 1983] O. Roldán, Tilted algebras of types $\widetilde{A_{n}}, \widetilde{B_{n}}, \widetilde{C_{n}}, \widetilde{B C_{n}}$, Ph.D. thesis, Carleton University, 1983.

[Schiffler 2008] R. Schiffler, "A geometric model for cluster categories of type $D_{n}$ ", J. Algebraic Combin. 27:1 (2008), 1-21. MR 2008k:16025 Zbl 1165.16008

[Schröer 1999] J. Schröer, "Modules without self-extensions over gentle algebras”, J. Algebra 216:1 (1999), 178-189. MR 2000d:16022 Zbl 0994.16013

[Schröer and Zimmermann 2003] J. Schröer and A. Zimmermann, "Stable endomorphism algebras of modules over special biserial algebras", Math. Z. 244:3 (2003), 515-530. MR 2004i:16024 Zbl 1036.16004

[Skowroński 1990] A. Skowroński, "Algebras of polynomial growth", pp. 535-568 in Topics in algebra, Part 1 (Warsaw, 1988), edited by S. Balcerzyk et al., Banach Center Publ. 26, PWN, Warsaw, 1990. MR 93k:16026 Zbl 0729.16005

Communicated by Andrei Zelevinsky

Received 2009-04-08 Revised 2009-06-24 Accepted 2009-08-06

ibrahim.assem@usherbrooke.ca

Département de Mathématiques, Université de Sherbrooke, Sherbrooke, QC J1K2R1, Canada

thomas.brustle@usherbrooke.ca Département de Mathématiques, Université de Sherbrooke, Sherbrooke, QC J1K2R1, Canada

Department of Mathematics, Bishop's University,

College St., Sherbrooke, QC J1M0C8, Canada

gabrielle.charbonneau-jodoin@usherbrooke.ca

Département de Mathématiques, Université de Sherbrooke, Sherbrooke, QC J1K2R1, Canada

plamondon@math.jussieu.fr

Institut de Mathématiques,

Université Denis Diderot (Paris VII),

Case 7012-2, place Jussieu 75251 Paris Cedex 05, France 